\title{
Reduction of Distributions: Definitions, Properties and Applications
}

\author{
Liyong Lin, Simon Ware, Rong Su, and W. Murray Wonham
}

Version Post-Print/Accepted Manuscript

Citation L. Lin; S. Ware; R. Su; W. M. Wonham, "Reduction of Distributions:

(published version) Definitions, Properties and Applications," in IEEE Transactions on Automatic Control, vol.PP, no.99, pp.1-1, doi:

10.1109/TAC.2017.2692561

Publisher's Statement $\quad$ C (C) 2017 IEEE. Personal use of this material is permitted. Permission from IEEE must be obtained for all other uses, in any current or future media, including reprinting/republishing this material for advertising or promotional purposes, creating new collective works, for resale or redistribution to servers or lists, or reuse of any copyrighted component of this work in other works.

Always cite the published version, so the author(s) will receive recognition through services that track citation counts, e.g. Scopus. If you need to cite the page number of the TSpace version (original manuscript or accepted manuscript) because you cannot access the published version, then cite the TSpace version in addition to the published version using the permanent URI (handle) found on the record page. 


\title{
Reduction of Distributions: Definitions, Properties and Applications
}

\author{
Liyong Lin, Simon Ware, Rong Su, W. Murray Wonham
}

\begin{abstract}
In this work, a notion of reduction of distributions is proposed as a technical tool for improving the complexity of decomposability verification and supporting parallel verification of decomposability, by exploiting the rich structures of distributions. We provide some results that reduce the search space of candidate reductions, as a first step towards efficiently computing optimal reductions. It is then shown that a distribution has a reduction if and only if a particular candidate reduction is indeed a reduction. We then provide a sound substitution-based proof technique that can be used for (automatic) reduction verification. Techniques for refuting candidate reductions are also provided. We then explain an application of the decomposability verification problem in the lower bound proofs for the problem of supervisor decomposition and the problem of existence of decentralized supervisor. Finally, some other applications of the notion of reduction of distributions are also shown.
\end{abstract}

Index Terms - discrete-event systems, decentralized supervisor synthesis, decomposability, complexity

\section{INTRODUCTION}

A language $L \subseteq \Sigma^{*}$ is said to be decomposable with respect to a distribution, i.e., a tuple of non-empty sub-alphabets, of $\Sigma$ if $L$ is equal to the synchronous product of its projections onto the respective sub-alphabets. The notion of decomposability is well studied and applied in the literature on supervisory control theory, see for example [1], [2], [3], [4], [5], [6], and synthesis of synchronous products of transition systems [7]. It is known that decomposability is a special case of co-observability [6].

In this work, we propose a structural approach for improving the complexity of decomposability verification and supporting parallel verification of decomposability, based on the notion of reduction of distributions, which is inspired by and generalizes a recent result on polynomial time verification of conditional decomposability in [3]. Intuitively, a reduction of a distribution is simply a set of smaller sized distributions with the property that, for an arbitrary language $L$ over $\Sigma$, it is decomposable with respect to each smaller sized distribution in the reduction if and only if it is decomposable with respect to the original distribution. To verify the decomposability of $L$ with respect to the given distribution, we can now verify the decomposability of $L$ with respect to each smaller sized distribution. The main motivation for our research on the decomposability verification problem is its close relationship with the problems of existence and synthesis of decentralized supervisor [5], [8]. In particular, a structural approach for improving the complexity of decomposability verification can lead to a better understanding of the decidability and complexity of the problems of existence and synthesis of decentralized supervisor, in relation to the structures of the underlying decentralized control architectures [9], which still remains largely unexplored.

The main contributions of this study are the following.

1) We propose a notion of reduction of distributions, which could be used to improve the complexity of decomposability verification and supports the parallel verification

L. Lin and W. M. Wonham are affiliated with Department of Electrical and Computer Engineering, University of Toronto. S. Ware and R. Su are with the School of Electrical and Electronic Engineering, Nanyang Technological University, Singapore. Email: liyong. I indutoronto.ca of decomposability for a large class of distributions. We provide results that reduce the search space of candidate reductions, as a first step towards efficiently computing the optimal reductions. We then show that a distribution does have a reduction if and only if a particular candidate reduction is indeed a reduction of the distribution. The problem of determining the existence of a reduction is therefore reduced to verifying that particular candidate reduction. We provide a sound substitution-based proof technique that can be used for reduction verification. On the other hand, several techniques for refuting candidate reductions are also provided, which could then be used to prove the following two kinds of results: a) a given distribution does not have any reduction; and b) a given reduction is compact, i.e., involves no redundancy.

2) We explain an application of the decomposability verification problem in the lower bound proofs for the problem of supervisor decomposition and also the problem of existence of decentralized supervisor.

3) For some other applications of the notion of reduction, we address the problem of determining the existence of a non-empty decomposable sublanguage and the problem of computing the infimal decomposable superlanguage, with respect to multiple distributions. Its applications to these two theoretical problems, which remain conceptually difficult, illustrate the surprising power of the notion of reduction.

This work provides an interesting generalization of the result on polynomial time verification of conditional decomposability (see Theorem 8 of [3]), and now the structures of distributions are not restricted to the case where the intersections of every two distinct sub-alphabets are equal, which is required by the definition of conditional decomposability. Indeed, structures of distributions can now be exploited automatically by computing reductions. Several basic results and techniques are developed for the automatic verification of reductions and computation of (optimal) reductions, which essentially could be viewed as automated theorem proving ${ }^{1}$ in the context of decomposability. Automated theorem proving in this very restricted setting relies on a sound substitution-based proof technique, which utilizes Lemma 3 in Section II as the only rule of inference. This work could also be viewed as a continuation of the systematic study on how the information structure determines the decidability and complexity of several verification and synthesis problems in decentralized supervisory control [8], [9]. This work is an extension of the short conference paper [10]. We have included in this paper all the proofs, an appendix on decomposability verification for prefix-closed regular languages and a summary about the substitution-based proof technique, e.t.c.

The paper is organized as follows. Section II is devoted to

\footnotetext{
${ }^{1}$ Indeed, verifying a set of smaller sized distributions to be a reduction of a given distribution is equivalent to proving the theorem/proposition that for any language $L, L$ is decomposable with respect to each smaller sized distribution if and only if $L$ is decomposable with respect to the given distribution.
} 
preliminaries. In Section III-A, we present the main definition. Some basic but important properties and results are presented in Section III-B. Some reductions are provided in Section IV for two special structures of distributions, on which the sound substitution-based proof technique for reduction verification is illustrated. The application of the decomposability verification problem in the lower bound proofs is provided in Section V. Some other interesting applications of the notion of reduction are shown in Section VI. We then draw conclusions in the last section, Section VII. In the Appendix, we provide an alternative algorithm for decomposability verification, which assumes prefix-closedness of test languages, and then compares it with the reduction-based approach.

\section{PRELIMINARIES}

We assume that the readers are familiar with basic theories of formal languages and finite automata [11]. Some knowledge about order theory [12], [13] is also assumed. In the following, additional notations and terminologies are introduced.

Let $[1, n]$ denote the set $\{1,2, \ldots, n\}$. For any two sets $A$ and $B$, we write $A-B$ to denote the set-theoretic difference of $A$ and $B$. The cardinality of any set $A$ is denoted by $|A|$. For any given alphabet $\Sigma$, a distribution of $\Sigma$ of size $n$ is an $n$-tuple $\Delta=\left(\Sigma_{1}, \Sigma_{2}, \ldots, \Sigma_{n}\right)$ of non-empty sub-alphabets of $\Sigma$ such that $\Sigma=\bigcup_{i=1}^{n} \Sigma_{i}$ and the sub-alphabets are pairwise incomparable with respect to set inclusion. We sometimes also view the distribution $\Delta$ as the set $\left\{\Sigma_{1}, \Sigma_{2}, \ldots, \Sigma_{n}\right\}$ for convenience and use $|\Delta|$ to denote the size of $\Delta$. Given a distribution $\Delta=\left(\Sigma_{1}, \Sigma_{2}, \ldots, \Sigma_{n}\right)$ of $\Sigma$, we have $n$ projections $P_{i}$ from $\Sigma^{*}$ to $\Sigma_{i}^{*}$ and $n$ inverse projections $P_{i}^{-1}$ from $\Sigma_{i}^{*}$ to $2^{\Sigma^{*}}$. Also, both projections and inverse projections are naturally extended to the mappings between languages. The synchronous product $\|_{i=1}^{n} L_{i}$ of languages $L_{i}$ over $\Sigma_{i}$ is defined as $\bigcap_{i=1}^{n} P_{i}^{-1}\left(L_{i}\right)$. A language $L \subseteq \Sigma^{*}$ is said to be decomposable with respect to distribution $\Delta=\left(\Sigma_{1}, \Sigma_{2}, \ldots, \Sigma_{n}\right)$ of $\Sigma$ if $L=\|_{i=1}^{n} P_{i}(L)$. Let

$$
\begin{gathered}
s_{1} \amalg s_{2}=\left\{v_{1} u_{1} v_{2} u_{2} \ldots v_{k} u_{k} \mid s_{1}=v_{1} v_{2} \ldots v_{k}, s_{2}=\right. \\
\left.u_{1} u_{2} \ldots u_{k}, \text { where } v_{i}, u_{i} \in \Sigma^{*} \text { for each } i \in[1, k]\right\}
\end{gathered}
$$

denote the shuffle of any two strings $s_{1}, s_{2} \in \Sigma^{*}$, and similarly we let $L_{1} \amalg L_{2}=\bigcup_{s_{1} \in L_{1}, s_{2} \in L_{2}} s_{1} \amalg s_{2}$ denote the shuffle of any two languages $L_{1}, L_{2} \subseteq \Sigma^{*}$. For example, the shuffle of strings $s_{1}=a b$ and $s_{2}=a$ is $s_{1} \amalg s_{2}=\{a b a, a a b\}$. For any language $L_{i}$ over $\Sigma_{i}$, we have $P_{i}^{-1}\left(L_{i}\right)=L_{i} \amalg\left(\Sigma-\Sigma_{i}\right)^{*}$.

Each distribution $\Delta=\left(\Sigma_{1}, \Sigma_{2}, \ldots, \Sigma_{n}\right)$ induces a dependence relation $D_{\Delta} \subseteq \Sigma \times \Sigma$, which is defined below.

$$
D_{\Delta}=\left\{(a, b) \in \Sigma \times \Sigma \mid \exists i \in[1, n], a, b \in \Sigma_{i}\right\}
$$

Clearly, $D_{\Delta}$ is reflexive and symmetric. Then the complement $I_{\Delta}=\Sigma \times \Sigma-D_{\Delta}$ of $D_{\Delta}$ is said to be the independence relation induced by $\Delta$, which is irreflexive and symmetric. For any $(a, b) \in I_{\Delta}, a$ and $b$ are said to be independent symbols. Two strings $s, s^{\prime}$ over $\Sigma$ are said to be trace equivalent with respect to the independence relation $I_{\Delta}$, denoted by $s \sim_{I_{\Delta}} s^{\prime}$, if there exist strings $v_{0}, \ldots, v_{k}$ such that $s=v_{0}, s^{\prime}=v_{k}$ and for each $i \in[1, k]$, there exist some $u_{i}, u_{i}^{\prime} \in \Sigma^{*}$ and $a_{i}, b_{i} \in \Sigma$ such that $\left(a_{i}, b_{i}\right) \in I_{\Delta}, v_{i-1}=u_{i} a_{i} b_{i} u_{i}^{\prime}$ and $v_{i}=u_{i} b_{i} a_{i} u_{i}^{\prime}$. Intuitively, two strings are trace equivalent if each one of them can be obtained from the other by a sequence of permutations of adjacent symbols that are independent. And the set of trace equivalent strings of $s$ for $I_{\Delta}$ is said to be the trace closure of $s$ with respect to $I_{\Delta}$, denoted by $[s]_{I_{\Delta}}$. The trace closure $[L]_{I_{\Delta}}$ of a language $L \subseteq \Sigma^{*}$ is defined to be the set $\bigcup_{s \in L}[s]_{I_{\Delta}}$. A language $L$ is said to be trace-closed with respect to $I_{\Delta}$ if $L=$ $[L]_{I_{\Delta}}$. For example, consider the independence relation $I_{\Delta}=$ $\{(a, c),(c, a),(b, c),(c, b)\}$ induced by $\Delta=(\{a, b\},\{c\})$. The strings $b a c, c b a$ are trace equivalent with respect to $I_{\Delta}$ since $b a c \sim_{I_{\Delta}} b c a \sim_{I_{\Delta}} c b a . L=\{b a c, c b a, b c a\}$ is trace-closed with respect to $I_{\Delta}$ since we have $[L]_{I_{\Delta}}=L=\{b a c, c b a, b c a\}$. An independence relation $I_{\Delta} \subseteq \Sigma \times \Sigma$ is said to be transitive, if for all $a, b, c \in \Sigma, a \neq c,(a, b) \in I_{\Delta},(b, c) \in I_{\Delta}$ implies $(a, c) \in I_{\Delta}$.

The prefix closure of a language $L$ is denoted by $\bar{L}$ and the complementation of $L$ is denoted by $L^{c}$. We write $\operatorname{Reg}(\Sigma)$ to denote the class of regular languages over $\Sigma$. A deterministic finite automaton $G=\left(Q, \Sigma, \delta, q_{0}, Q_{m}\right)$, where $Q$ is the finite state set, $\Sigma$ the alphabet, $\delta: Q \times \Sigma \longrightarrow Q$ the partial transition function, $q_{0} \in Q$ the initial state, $Q_{m} \subseteq Q$ the set of marked states, is said to be non-marking if each state of $G$ is a marked state, that is, if $Q_{m}=Q$. As usual, $\delta$ can be naturally extended to the partial transition function $\delta: Q \times \Sigma^{*} \longrightarrow Q \cdot|Q|$ is said to be the state size of $G$. We define $L(G)=\left\{s \in \Sigma^{*} \mid\right.$ $\delta\left(q_{0}, s\right)$ is defined. $\}$. And we say a regular language $L$ is given by a deterministic finite automaton $G$ if the marked behavior $L_{m}(G)=\left\{s \in \Sigma^{*} \mid s \in L(G), \delta\left(q_{0}, s\right) \in Q_{m}\right\}$ of $G$ is equal to $L$. We often do not distinguish between the string $s$ and the singleton $\{s\}$.

We need to use the following well established results (see Exercise 3.3.7 in [13] for a statement ${ }^{2}$ of Lemma 3 for $n=2$, and see [9] and the references therein for Lemma 2).

Lemma 1. Given any distribution $\Delta$ of $\Sigma$ and any language $L$ over $\Sigma, L \subseteq \|_{i=1}^{n} P_{i}(L)$.

Lemma 2. Given any distribution $\Delta$ of $\Sigma$ and any language $L$ over $\Sigma$. If $L$ is decomposable with respect to $\Delta$, then $L$ is trace-closed with respect to $I_{\Delta}$.

Lemma 3. Let $L_{i} \subseteq \Sigma_{i}^{*}$ for $i \in[1, n]$ and $\Sigma=\bigcup_{i=1}^{n} \Sigma_{i}$. If $\bigcup_{i \neq j}\left(\Sigma_{i} \cap \Sigma_{j}\right) \subseteq \Sigma_{0} \subseteq \Sigma$, then $P_{0}\left(\|_{i=1}^{n} L_{i}\right)=\|_{i=1}^{n} P_{0}\left(L_{i}\right)$. Here $P_{0}: \Sigma^{*} \mapsto \Sigma_{0}^{*}$ is the natural projection.

Remark 4. It is important to note that $P_{0}\left(L_{i}\right) \subseteq\left(\Sigma_{0} \cap \Sigma_{i}\right)^{*}$ is viewed as a language over $\Sigma_{0} \cap \Sigma_{i}$ in the above equality, instead of being viewed as a language over $\Sigma_{0}$. We note that a mathematically rigorous view, which is not used in this work, is as follows. In this special case, the restriction of $P_{0}: \Sigma^{*} \mapsto$ $\Sigma_{0}^{*}$ to the domain $\Sigma_{i}^{*}$ and co-domain $\left(\Sigma_{0} \cap \Sigma_{i}\right)^{*}$ is viewed as the projection $P_{0}: \Sigma_{i}^{*} \mapsto\left(\Sigma_{0} \cap \Sigma_{i}\right)^{*}$. It is sometimes desirable to use $Q_{i}$ to denote the projection $P_{0}: \Sigma_{i}^{*} \mapsto\left(\Sigma_{0} \cap \Sigma_{i}\right)^{*}$, for each $i \in[1, n]$. Then, the above equality can be rewritten as $P_{0}\left(\|_{i=1}^{n} L_{i}\right)=\|_{i=1}^{n} Q_{i}\left(L_{i}\right)$ (see also Proposition 4.3 in [14]).

The next result will also be useful.

Lemma 5. Let $\left\{\Delta_{1}, \Delta_{2}, \ldots, \Delta_{n}\right\}$ be any set of distributions of $\Sigma$. If $L$ is trace-closed with respect to each $I_{\Delta_{i}}$, then $L$ is trace-closed with respect to $\bigcup_{i=1}^{n} I_{\Delta_{i}}$.

Proof: By definition, the trace closure $[L]_{\bigcup_{i=1}^{n} I_{\Delta_{i}}}$ of $L$ with respect to the independence relation $\bigcup_{i=1}^{n} I_{\Delta_{i}}$ corresponds to the unique supremal element of the ascending chain

$$
\begin{gathered}
L \subseteq[L]_{I_{\Delta_{i}}} \subseteq\left[[L]_{I_{\Delta_{1}}}\right]_{I_{\Delta_{2}}} \subseteq \ldots \subseteq\left[\left[[L]_{I_{\Delta_{1}}}\right]_{I_{\Delta_{2}}} \ldots\right]_{I_{\Delta_{n}}} \subseteq \\
{\left[\left[\left[[L]_{I_{\Delta_{1}}}\right]_{I_{\Delta_{2}}} \ldots\right]_{I_{\Delta_{n}}}\right]_{I_{\Delta_{1}}} \subseteq \ldots}
\end{gathered}
$$

${ }^{2}$ See also Proposition 4.3 in [14], where a detailed proof is given. 
If $L$ is trace-closed with respect to each $I_{\Delta_{i}}$, i.e., $[L]_{I_{\Delta_{i}}}=L$ for each $i \in[1, n]$, then we conclude that each term in the ascending chain is indeed equal to $L$. Thus, $[L]_{\bigcup_{i=1}^{n} I_{\Delta_{i}}}=L$, i.e., $L$ is trace-closed with respect to $\bigcup_{i=1}^{n} I_{\Delta_{i}}$.

\section{REDUCTION OF DistRIBUTIONS}

Recall that a language $L$ over $\Sigma$ is said to be decomposable with respect to distribution $\Delta=\left(\Sigma_{1}, \Sigma_{2}, \ldots, \Sigma_{n}\right)$ of $\Sigma$ if $L=$ $\|_{i=1}^{n} P_{i}(L)$. For an arbitrary regular language $L \subseteq \Sigma^{*}$ that is given by a deterministic finite automaton of state size $m$, the straightforward approach for checking the decomposability of $L$ with respect to $\Delta$ is of worst case time complexity ${ }^{3} \mathcal{O}(((n+$ 1) $\left.\left.|\Sigma|-\sum_{i=1}^{n}\left|\Sigma_{i}\right|\right) m^{n+1}\right)$ [1], [3], which is exponential with respect to the size of $\Delta$. It is quite unlikely that a polynomial time decomposability verification algorithm would exist, for it is known that this verification problem is PSPACE-complete, even when $L$ is required to be prefix-closed (see Theorem 4.24 in [7]).

Recently, it was shown in [3] that there is an algorithm for decomposability verification with time complexity $\mathcal{O}\left(n|\Sigma| m^{3}\right)$ when distribution $\Delta$ possesses Property $C$ ):

$$
\exists \Sigma_{0} \subseteq \Sigma, \forall i, j \in[1, n],\left(i \neq j \Longrightarrow \Sigma_{i} \cap \Sigma_{j}=\Sigma_{0}\right)
$$

In particular, the following interesting observation was made: when Property $C$ ) holds for $\Delta$, an arbitrary language $L$ over $\Sigma$ is decomposable with respect to $\Delta=\left(\Sigma_{1}, \Sigma_{2}, \ldots, \Sigma_{n}\right)$ if and only if it is decomposable with respect to $\Delta_{i}=\left(\Sigma_{i}, \bigcup_{j \neq i} \Sigma_{j}\right)$ for each $i \in[1, n]$, that is, verifying the decomposability of $L$ with respect to $\Delta$ is reduced to verifying the decomposability of $L$ with respect to distributions $\Delta_{i}$ 's. Since each distribution $\Delta_{i}$ is of size 2 , it is then possible to verify the decomposability of $L$ with respect to each distribution $\Delta_{i}$ in $\mathcal{O}\left(|\Sigma| m^{3}\right)$ time using the algorithm of [1], [3], and thus it becomes possible to verify the decomposability of $L$ with respect to distribution $\Delta$ in $\mathcal{O}\left(n|\Sigma| m^{3}\right)$ time. It turns out that variations of the above observation also hold for a much wider class of distributions. In general, for an arbitrary given distribution $\Delta$ of $\Sigma$, we hope to find a set $\left\{\Delta_{1}, \Delta_{2}, \ldots, \Delta_{l}\right\}$ of smaller sized distributions so that verifying the decomposability of any language $L$ with respect to $\Delta$ is reduced to verifying the decomposability of $L$ with respect to distributions $\Delta_{i}$ 's. Indeed, the verification and computation of such a set $\left\{\Delta_{1}, \Delta_{2}, \ldots, \Delta_{l}\right\}$ of smaller sized distributions, which is referred to as a reduction of $\Delta$, will be the main task of this work. The problem of computing reductions is of interest at least for the following reasons: 1) they provide complexity reduction for decomposability verification, and also parallel verification ${ }^{4}$ of decomposability can be easily supported using these results; 2) other efficient techniques for decomposability verification and language inclusion checking ${ }^{5}$ may be integrated, as verifications of the decomposability of $L$ with respect to these $\Delta_{i}$ 's still need to be carried out and could be optimized accordingly; 3 ) each reduction $\Delta_{i}$ 's works for any language $L \subseteq \Sigma^{*}$ and thus it might be useful to compute such reductions offline and then store them for potential future usage, e.g., when different languages need to be tested for their

\footnotetext{
${ }^{3}$ Step 2) of Algorithm 4.1 in [1] has to be eliminated. Otherwise, the time complexity of the given algorithm is $\mathcal{O}\left(\left(\sum_{\sigma \in \Sigma} m^{\left|I_{\sigma}\right|}\right) m^{n+1}\right)$, where $I_{\sigma}=$ $\left\{i \in[1, n] \mid \sigma \in \Sigma_{i}\right\}$ for each $\sigma \in \Sigma$.

${ }^{4}$ The tasks of verifying the decomposability of $L$ with respect to individual distributions $\Delta_{1}, \Delta_{2}, \ldots, \Delta_{l}$ can be carried out in parallel.

${ }^{5}$ We only need to check the language inclusion $\|_{i=1}^{n} P_{i}(L) \subseteq L$ to verify decomposability, by Lemma 1 .
}

decomposability with respect to the same distribution $\Delta$; and 4) they could provide some insights into the boundary between the tractable and intractable fragments of the decomposability verification problem, and also some other related verification problems in the realm of decentralized supervisory control, as we shall explain in details in Section V. The general approach based on computing reductions is appealing, since it provides a systematic method for studying structures of distributions to reduce decomposability verification complexity. The reader is referred to Section VI for other applications of this approach.

We are heavily motivated by the following list of long term questions.

a) What structural conditions shall a distribution satisfy for it to have a reduction?

b) For any given distribution, how to efficiently determine whether it has a reduction and, if it has a reduction, how to efficiently compute an (optimal) reduction?

c) how powerful is the reduction-based approach, in other words, which tractable fragments of the decomposability verification problem could be identified using the notion of reduction?

A formal definition of the notion of reduction (of distributions) and some other related concepts shall be provided in the next subsection.

\section{A. Main Definition}

We propose the following definition to formally capture the main idea of our reduction-based approach.

Definition 6. A set $\left\{\Delta_{1}, \Delta_{2}, \ldots, \Delta_{l}\right\}$ of distributions is said to be a reduction of $\Delta$ if the following conditions hold: 1) an arbitrary language $L \subseteq \Sigma^{*}$ is decomposable with respect to $\Delta_{i}$ for each $i \in[1, l]$ if and only if it is decomposable with respect to $\Delta$; 2) for each $i \in[1, l],\left|\Delta_{i}\right|<|\Delta|$.

Remark 7. Clearly, 1) is the main condition of Definition 6. Condition 2) is added so that the complexity of verifying the decomposability of $L$ with respect to each $\Delta_{i}$ is lower than the complexity of the verification with respect to $\Delta$, using the algorithm of [1], [3]. We remark here in advance that although Condition 1) is required to hold for every language over $\Sigma$, including all the non-regular languages, it is sufficient to only require $L$ to be regular ${ }^{6}$.

Remark 8. Let $\left\langle\Delta_{1}, \Delta_{2}, \ldots, \Delta_{l}\right\rangle \subseteq \boldsymbol{R} \operatorname{eg}(\Sigma)$ denote the decision problem ${ }^{7}$ of verifying the decomposability of an arbitrary regular language with respect to distributions $\Delta_{1}, \Delta_{2}, \ldots, \Delta_{l}$, that is, we use $\left\langle\Delta_{1}, \Delta_{2}, \ldots, \Delta_{l}\right\rangle$ to denote the set of regular languages over $\Sigma$ that are decomposable with respect to the

\footnotetext{
${ }^{6}$ Indeed, it turns out to be sufficient to require $L$ to be a finite language! This will be shown in Proposition 29 and Corollary 30 in Section III-B.

${ }^{7}$ Using the terminology of descriptive complexity [15], a decision problem is just a set STRUC $[\sigma]$ of structures of a given vocabulary $\sigma$. Given any fixed alphabet $\Sigma$, a deterministic finite automaton over $\Sigma$ is simply a structure $G=$ $\left(Q, q_{0}^{Q}, Q_{m}^{Q},\left(\delta_{\sigma}^{Q}\right)_{\sigma \in \Sigma}\right)$ of the vocabulary $\sigma_{d f a}=\left(q_{0} ; Q_{m}^{1},\left(\delta_{\sigma}^{2}\right)_{\sigma \in \Sigma}\right)$. In $\sigma_{d f a}, q_{0}$ is a constant symbol for the initial state, $Q_{m}$ is a relational symbol (of arity 1 ) for the set of marked states and each $\delta_{\sigma}$ is a relational symbol (of arity 2) for the transition relation associated with event $\sigma$. Then, formally,

$$
\left\langle\Delta_{1}, \Delta_{2}, \ldots, \Delta_{l}\right\rangle=\left\{G \in \operatorname{STRUC}\left[\sigma_{d f a}\right] \mid G \models \bigwedge_{i=1}^{l} \operatorname{decom}\left(\Delta_{i}\right)\right\}
$$
}

where $\operatorname{decom}\left(\Delta_{i}\right)$ is a formula which specifies that $L_{m}(G)$ is decomposable with respect to $\Delta_{i}$ (the details are not important here). For convenience, we also write in language terms

$$
\begin{gathered}
\left\langle\Delta_{1}, \Delta_{2}, \ldots, \Delta_{l}\right\rangle=\left\{L_{m}(G) \mid G \in\right. \\
\left.\operatorname{STRUC}\left[\sigma_{d f a}\right], G \models \bigwedge_{i=1}^{l} \operatorname{decom}\left(\Delta_{i}\right)\right\} \subseteq \operatorname{Reg}(\Sigma)
\end{gathered}
$$


distributions $\Delta_{1}, \Delta_{2}, \ldots, \Delta_{l}$. Similarly, we let $\langle\Delta\rangle \subseteq \boldsymbol{R} \operatorname{eg}(\Sigma)$ denote the decision problem of verifying the decomposability of an arbitrary regular language with respect to $\Delta$. Suppose $\left|\Delta_{i}\right|<|\Delta|$ holds for each $i \in[1, l]$. We here shall note that 1) verifying a set of distributions $\left\{\Delta_{1}, \Delta_{2}, \ldots, \Delta_{l}\right\}$ to be a reduction of $\Delta$ is equivalent to verifying the identity function $f=i d$ to be a reduction ${ }^{8}$ from problem $\langle\Delta\rangle$ to problem $\left\langle\Delta_{1}, \Delta_{2}, \ldots, \Delta_{l}\right\rangle$; 2) computing a reduction of distribution $\Delta$ is then equivalent to searching for a decision problem of the form $\left\langle\Delta_{1}, \Delta_{2}, \ldots, \Delta_{l}\right\rangle$ to which $\langle\Delta\rangle$ can be reduced using the identity function. The problem of computing reductions of a distribution has to be distinguished from that of computing reductions between (the above) decision problems [15], where $f$ is not restricted to being the identity function and needs to be computed if it exists. It is not at all obvious whether there exists an efficiently computable (e.g., linear time computable) non-identity function $f$ that renders a more powerful notion of reduction of distributions.

$\Delta$ is said to be reducible if it has a reduction. $\Delta$ is said to be $(l, k)$-reducible if it has a reduction $\left\{\Delta_{1}, \Delta_{2}, \ldots, \Delta_{l}\right\}$, where $\max _{i \in[1, l]}\left|\Delta_{i}\right|=k$; and this reduction will be referred to as an $(l, k)$-reduction of $\Delta$, where $l$ is called the height, $k$ the width and $(l, k)$ the dimension (of the reduction). Rephrased in our terminology, $\Delta=\left(\Sigma_{1}, \Sigma_{2}, \ldots, \Sigma_{n}\right)$ is $(n, 2)$-reducible if Property $C)$ holds; and then $\left\{\left(\Sigma_{i}, \bigcup_{j \neq i} \Sigma_{j}\right) \mid i \in[1, n]\right\}$ is an $(n, 2)$-reduction of $\Delta$. A reduction may involve unnecessary redundancy. For example, it could be shown that any subset of the reduction $\left\{\left(\Sigma_{i}, \bigcup_{j \neq i} \Sigma_{j}\right) \mid i \in[1, n]\right\}$ of cardinality $n-1$ is an $(n-1,2)$-reduction of $\Delta$, if Property $C$ ) holds ${ }^{9}$ for $\Delta$. A reduction of $\Delta$ is said to be compact if none of its proper subsets is also a reduction of $\Delta$. Ideally, we would always like a reduction to be compact. Unfortunately, verifying compactness requires refuting all the proper subsets of a reduction and is not always easy to achieve. A weaker requirement will be used to eliminate some obvious redundancy. In general, a distribution may have compact reductions of different dimensions and also different compact reductions of the same dimension. This shall be illustrated by the following example.

Example 9. Consider the distribution $\Delta=\left(\Sigma_{1}, \Sigma_{2}, \Sigma_{3}, \Sigma_{4}\right)$ of $\Sigma=\{a, b, c, d, e, f\}$, where $\Sigma_{1}=\{a, b\}, \Sigma_{2}=\{b, c\}$, $\Sigma_{3}=\{d, e\}$ and $\Sigma_{4}=\{e, f\}$. It is possible to show, using a technique to be demonstrated later in Section IV, that the set $\left\{\Delta_{1}, \Delta_{2}\right\}$ of distributions is a (2,3)-reduction of $\Delta$, where

$$
\left\{\begin{aligned}
\Delta_{1} & =(\{a, b\},\{b, c\},\{d, e, f\}) \\
\Delta_{2} & =(\{d, e\},\{e, f\},\{a, b, c\})
\end{aligned}\right.
$$

and $\left\{\Delta_{1}^{\prime}, \Delta_{2}^{\prime}, \Delta_{3}^{\prime}\right\}$ is a $(3,2)$-reduction of $\Delta$, where

$$
\left\{\begin{aligned}
\Delta_{1}^{\prime} & =(\{a, b\},\{b, c, d, e, f\}) \\
\Delta_{2}^{\prime} & =(\{a, b, c\},\{d, e, f\}) \\
\Delta_{3}^{\prime} & =(\{d, e\},\{a, b, c, e, f\})
\end{aligned}\right.
$$

and $\left\{\Delta_{1}^{\prime \prime}, \Delta_{2}^{\prime \prime}\right\}$ is a $(2,2)$-reduction of $\Delta$, where

\footnotetext{
${ }^{8}$ As discussed in Section 2.6 of [15], given any pair of decision problems $\mathcal{S} \subseteq \operatorname{STRUC}[\sigma]$ and $\mathcal{T} \subseteq \operatorname{STRUC}[\tau]$, a many-one reduction from $\mathcal{S}$ to $\mathcal{T}$ is an "easy-to-compute" function $f: \operatorname{STRUC}[\sigma] \longrightarrow \operatorname{STRUC}[\tau]$ such that for every $A \in \operatorname{STRUC}[\sigma]$,

$A \in S$ if and only if $f(A) \in T$

First order reductions are used in [15]. Here we deal with the simpler setting where $\sigma=\tau=\sigma_{d f a}$ and $f$ is the trivial identity function $i d$, that is, for any $G \in \operatorname{STRUC}\left[\sigma_{d f a}\right], f(G)=i d(G)=G$. In language terms, we have $f(L)=i d(L)=L$ for any regular language $L \in \operatorname{Reg}(\Sigma)$.

${ }^{9}$ See Proposition 32 for a generalization.
}

$$
\left\{\begin{array}{l}
\Delta_{1}^{\prime \prime}=(\{a, b, d, e\},\{b, c, e, f\}) \\
\Delta_{2}^{\prime \prime}=(\{a, b, c\},\{d, e, f\})
\end{array}\right.
$$

and $\left\{\Delta_{1}^{\prime \prime \prime}, \Delta_{2}^{\prime \prime \prime}\right\}$ is another $(2,2)$-reduction of $\Delta$, where

$$
\left\{\begin{array}{l}
\Delta_{1}^{\prime \prime \prime}=(\{a, b, e, f\},\{b, c, d, e\}) \\
\Delta_{2}^{\prime \prime \prime}=(\{a, b, c\},\{d, e, f\})
\end{array}\right.
$$

Clearly, reductions $\left\{\Delta_{1}, \Delta_{2}\right\},\left\{\Delta_{1}^{\prime \prime}, \Delta_{2}^{\prime \prime}\right\}$ and $\left\{\Delta_{1}^{\prime \prime \prime}, \Delta_{2}^{\prime \prime \prime}\right\}$ are compact, since their heights are exactly two ${ }^{10}$. It will be shown later that $\left\{\Delta_{1}^{\prime}, \Delta_{2}^{\prime}, \Delta_{3}^{\prime}\right\}$ is also a compact reduction.

Remark 10. Let $\Delta$ be any distribution of $\Sigma$ of size $n$. If $\Delta$ has an $(l, k)$-reduction, then the verification of the decomposability of $L$ with respect to $\Delta$ costs $\mathcal{O}\left(l k|\Sigma| m^{k+1}\right)$ time, where $k<n$. Here we have used the fact that a crude upper bound for the time complexity of the decomposability verification algorithm in [1], [3] is $\mathcal{O}\left(k|\Sigma| m^{k+1}\right)$ for any distribution of size at most $k$. Thus, to ensure complexity improvement using the reduction $\left\{\Delta_{1}, \Delta_{2}, \ldots, \Delta_{l}\right\}, l$ needs to be relatively small and we could impose an extra condition on reductions: $l \leq|\Delta|^{d}$ for some small natural number $d \in \mathbb{N}$, e.g., $d \leq 2$ (that is, the number of distributions in the reduction is at most quadratic in the size of $\Delta$ ). If, in addition, $l \leq|\Delta|^{d}$ for some small $d$, where $|\Delta|=n$, then the time complexity becomes $\mathcal{O}\left(n^{d} k|\Sigma| m^{k+1}\right)$, which is better than $\mathcal{O}\left(\left((n+1)|\Sigma|-\sum_{i=1}^{n}\left|\Sigma_{i}\right|\right) m^{n+1}\right)$, assuming the mild condition $n^{d} k \ll m^{n-k}$.

Remark 11. There is a technical reason why we could assume $d$ is a small natural number. From Theorem 1 to be established later, we know that if there exists a reduction of $\Delta$, then there exists an $(l, k)$-reduction of $\Delta$ with $l \leq|\Delta|(|\Delta|-1) / 2<|\Delta|^{2}$. Thus, a choice of $d=2$ would be enough if our purpose is to determine the existence of a reduction of $\Delta$. However, we are not sure whether all reductions' heights are bounded by $|\Delta|^{2}$, if we consider only those reductions that are compact.

Formally, we say a reduction $P$ of distribution $\Delta$ is optimal if $P$ is compact and for any other reduction $P^{\prime}$ of $\Delta$, the width of $P^{\prime}$ is greater than or equal to ${ }^{11}$ the width of $P$.

In the rest of this work, we shall develop results that could be used to address the following main technical problems.

1) given any distribution $\Delta$ and any set $P$ of distributions, determine whether $P$ is a reduction of $\Delta$

2) given any distribution $\Delta$ of $\Sigma$, determine whether $\Delta$ has a reduction

3) given any distribution $\Delta$ of $\Sigma$ and any reduction $P$ of $\Delta$, determine whether $P$ is compact

4) given any distribution $\Delta$, compute an (optimal) reduction, if there exists one

5) given any set $P$ of distributions, compute a distribution $\Delta$ such that $P$ is a reduction of $\Delta$, if there exists one

The first problem is the most important one among the above list of problems, since its resolution will lead to the resolution of the other problems, from the point of view of computability. We shall also develop properties and results in Section III-B that can facilitate fast verification and computation of reductions. We shall here remark that Technical Problem 5 is useful in the application to be discussed in Section VI-B.

\footnotetext{
${ }^{10}$ It will later be shown that the height of any reduction is at least two.

${ }^{11}$ This is only a working definition of optimality that serves for illustration purposes: we need to minimize the exponent $k$ in $\mathcal{O}\left(l k|\Sigma| m^{k+1}\right)$.
} 


\section{B. Basic Properties and Results}

This subsection is devoted to a detailed study of some basic properties of distributions, decomposability and reductions of distributions. Several useful results will also be proved in this subsection. As a first step, we need to study an order-theoretic structure of the set of distributions with respect to which any given language is decomposable. To that end, we shall define a relation $\leq_{\Sigma}$ on the set $\Delta(\Sigma)$ of all distributions of $\Sigma$ (lifting the partial ordering $\subseteq$ ) as follows.

Definition 12. Given any two distributions $\Delta=\left(\Sigma_{i}\right)_{i=1}^{n}$ and $\Delta^{\prime}=\left(\Sigma_{j}^{\prime}\right)_{j=1}^{l}$ of $\Sigma$, we define $\Delta \leq_{\Sigma} \Delta^{\prime}$ if there exists a function $f:[1, n] \mapsto[1, l]$ such that $\forall i \in[1, n], \Sigma_{i} \subseteq \Sigma_{f(i)}^{\prime}$. In other words, $\Delta \leq_{\Sigma} \Delta^{\prime}$ iff $\forall i \in[1, n], \exists j \in[1, l], \Sigma_{i} \subseteq \Sigma_{j}^{\prime}$.

It is not difficult to check that the relation $\leq_{\Sigma}$ is a partial ordering on $\Delta(\Sigma)$. We now show that the set of distributions, with respect to which any given language is decomposable, is an upward closed set with respect to the partial ordering $\leq_{\Sigma}$ (Lemma 13).

Lemma 13. Given any two distributions $\Delta \leq_{\Sigma} \Delta^{\prime}$ of $\Sigma$ and any language $L$ over $\Sigma$, if $L$ is decomposable with respect to $\Delta$, then $L$ is also decomposable with respect to $\Delta^{\prime}$.

Proof: Let $\Delta=\left(\Sigma_{1}, \Sigma_{2}, \ldots, \Sigma_{n}\right), \Delta^{\prime}=\left(\Sigma_{1}^{\prime}, \Sigma_{2}^{\prime}, \ldots, \Sigma_{l}^{\prime}\right)$. Suppose $L$ is decomposable with respect to $\Delta$. Since $\Delta \leq_{\Sigma}$ $\Delta^{\prime}$, by definition there exists a function $f:[1, n] \mapsto[1, l]$ such that $\forall i \in[1, n], \Sigma_{i} \subseteq \Sigma_{f(i)}^{\prime}$. Clearly, we have $\bigcup_{j \in f([1, n])} \Sigma_{j}^{\prime}=$ $\Sigma$. For each $j \in f([1, n])$ and for each $i \in f^{-1}(j)$, we have $P_{j}^{\prime-1} P_{j}^{\prime}(L) \subseteq P_{i}^{-1} P_{i}(L)$, since $\Sigma_{i} \subseteq \Sigma_{j}^{\prime}$. Thus, we conclude that $P_{j}^{\prime-1} P_{j}^{\prime}(L) \subseteq \bigcap_{i \in f^{-1}(j)} P_{i}^{-1} P_{i}(L)$. It follows that

$$
\bigcap_{j \in f([1, n])} P_{j}^{\prime-1} P_{j}^{\prime}(L) \subseteq \bigcap_{j \in f([1, n])} \bigcap_{i \in f^{-1}(j)} P_{i}^{-1} P_{i}(L)
$$

Clearly, we have $\bigcap_{j \in f([1, n])} \bigcap_{i \in f^{-1}(j)} P_{i}^{-1} P_{i}(L)=L$, since $\left\{f^{-1}(j) \mid j \in f([1, n])\right\}$ forms a partition of $[1, n]$ and $L$ is decomposable with respect to $\Delta$. It then follows that we have $\bigcap_{j \in f([1, n])} P_{j}^{\prime-1} P_{j}^{\prime}(L) \subseteq L$. We finally conclude that $L \subseteq \bigcap_{j \in[1, l]} P_{j}^{\prime-1} P_{j}^{\prime}(L) \subseteq \bigcap_{j \in f([1, n])} P_{j}^{\prime-1} P_{j}^{\prime}(L) \subseteq L$ and thus $L$ is decomposable with respect to $\Delta^{\prime}$.

Remark 14. For any language $L$ over $\Sigma$ and any distribution $\Delta=\left(\Sigma_{1}, \Sigma_{2}, \ldots, \Sigma_{n}\right)$ of $\Sigma$, let $L^{\Delta}$ denote $\|_{i=1}^{n} P_{i}(L)$. Then, Lemma 13 also follows from the fact that if $\Delta \leq_{\Sigma} \Delta^{\prime}$, then $L^{\Delta^{\prime}} \subseteq L^{\Delta}$, whose proof can be extracted from the proof of Lemma 13.

It turns out that the converse of Lemma 13 also holds.

Lemma 15. Given any two distributions $\Delta$ and $\Delta^{\prime}$ of $\Sigma$. If for every language $L \subseteq \Sigma^{*}$ the decomposability of $L$ with respect to $\Delta$ implies the decomposability of $L$ with respect to $\Delta^{\prime}$, then $\Delta \leq_{\Sigma} \Delta^{\prime}$.

Proof: Let $\Delta=\left(\Sigma_{1}, \Sigma_{2}, \ldots, \Sigma_{n}\right), \Delta^{\prime}=\left(\Sigma_{1}^{\prime}, \Sigma_{2}^{\prime}, \ldots, \Sigma_{l}^{\prime}\right)$. Suppose it is not the case that $\Delta \leq_{\Sigma} \Delta^{\prime}$. Then, there exists $i \in$ $[1, n]$ such that for each $j \in[1, l], \Sigma_{i} \not \Sigma_{j}^{\prime}$. Thus, $\Sigma_{i} \cap \Sigma_{j}^{\prime} \subset$ $\Sigma_{i}$ for each $j \in[1, l]$. Clearly, any language $L \subseteq \Sigma_{i}^{*}$, when viewed as ${ }^{12}$ a language over $\Sigma$, is decomposable with respect to $\Delta=\left(\Sigma_{1}, \Sigma_{2}, \ldots, \Sigma_{n}\right)$. Indeed, we first observe that each $P_{j}(L) \subseteq\left(\Sigma_{j} \cap \Sigma_{i}\right)^{*}$ is viewed as a language over $\Sigma_{j}$. Thus, for each $j, P_{j}^{-1}\left(P_{j}(L)\right)=P_{j}(L) \amalg\left(\Sigma-\Sigma_{j}\right)^{*} \subseteq\left(\Sigma-\left(\Sigma_{j}-\Sigma_{i}\right)\right)^{*}$.

\footnotetext{
${ }^{12}$ That is, no symbol in $\Sigma-\Sigma_{i}$ occurs in $L \subseteq \Sigma^{*}$
}

Thus, $\bigcap_{j \neq i} P_{j}^{-1}\left(P_{j}(L)\right) \subseteq \Sigma_{i}^{*}$. Then, we have, since $L \subseteq \Sigma_{i}^{*}$,

$$
\begin{gathered}
\|_{j=1}^{n} P_{j}(L)=\bigcap_{j \neq i} P_{j}^{-1}\left(P_{j}(L)\right) \cap P_{i}^{-1}\left(P_{i}(L)\right)= \\
\bigcap_{j \neq i} P_{j}^{-1}\left(P_{j}(L)\right) \cap P_{i}^{-1}(L)=
\end{gathered}
$$

$\bigcap_{j \neq i} P_{j}^{-1}\left(P_{j}(L)\right) \cap\left(L \amalg\left(\Sigma-\Sigma_{i}\right)^{*}\right)=\bigcap_{j \neq i} P_{j}^{-1}\left(P_{j}(L)\right) \cap L$

Since $L \subseteq P_{j}^{-1}\left(P_{j}(L)\right)$, for each $j \neq i$, we have $\|_{j=1}^{n} P_{j}(L)=$ $L$. Now we let $\Sigma_{i}$ be enumerated as $\left\{\sigma_{1}, \sigma_{2}, \ldots, \sigma_{m}\right\}=\left\{\sigma_{h} \mid\right.$ $h \in[1, m]\}$ for some integer $m$. It is not that difficult to check that the language $L=\left\{\sigma_{1}^{\delta_{1, j}} \sigma_{2}^{\delta_{2, j}} \ldots \sigma_{m}^{\delta_{m, j}} \mid j \in[1, l]\right\} \subseteq \Sigma_{i}^{*}$, when viewed as a language over $\Sigma$, is not decomposable with respect to $\Delta^{\prime}=\left(\Sigma_{1}^{\prime}, \Sigma_{2}^{\prime}, \ldots, \Sigma_{l}^{\prime}\right)$, where $\delta_{h, j}=1$ if $\sigma_{h} \in$ $\Sigma_{i} \cap \Sigma_{j}^{\prime}$ and $\delta_{h, j}=2$ otherwise. In fact, it can be shown that $s=\sigma_{1} \sigma_{2} \ldots \sigma_{m} \in \|_{j=1}^{l} P_{j}^{\prime}(L)$ but $s \notin L$. The reasoning is presented as follows.

From $\Sigma_{i} \cap \Sigma_{j}^{\prime} \subset \Sigma_{i}$, it follows that for each $j \in[1, l]$ there exists an integer $h \in[1, m]$ such that $\sigma_{h} \notin \Sigma_{i} \cap \Sigma_{j}^{\prime}$, that is, for each $j \in[1, l]$ there exists an integer $h \in[1, m]$ such that $\delta_{h, j}=2$. This implies that $s=\sigma_{1} \sigma_{2} \ldots \sigma_{m} \notin L$. Since $L \subseteq \Sigma_{i}^{*}$, we have $P_{j}^{\prime}(L) \subseteq\left(\Sigma_{i} \cap \Sigma_{j}^{\prime}\right)^{*}$. Furthermore, we have $P_{j}^{\prime}\left(\sigma_{1} \sigma_{2} \ldots \sigma_{m}\right)=P_{j}^{\prime}\left(\sigma_{1}^{\delta_{1, j}} \sigma_{2}^{\delta_{2, j}} \ldots \sigma_{m}^{\delta_{m, j}}\right) \in P_{j}^{\prime}(L)$ for each $j \in[1, l]$, from the definition of $\delta_{h, j}$. It follows that $s \in\left\|_{j=1}^{l} P_{j}^{\prime}(s) \subseteq\right\|_{j=1}^{l} P_{j}^{\prime}(L)$.

Lemma 15 implies that for any reduction $\left\{\Delta_{1}, \Delta_{2}, \ldots, \Delta_{l}\right\}$ of $\Delta$, we must have ${ }^{13} l \geq 2$. Another immediate implication of Lemma 15 is the next corollary, which provides a necessary condition for a set $\left\{\Delta_{1}, \Delta_{2}, \ldots, \Delta_{l}\right\}$ of distributions to be a reduction of $\Delta$.

Corollary 16. Let $\left\{\Delta_{1}, \Delta_{2}, \ldots, \Delta_{l}\right\}$ be any reduction of $\Delta$. Then, for each $i \in[1, l]$, we have $\Delta \leq_{\Sigma} \Delta_{i}$.

Corollary 16 helps to reduce the search space for reductions. We shall introduce another notion to further reduce the search space. We say a distribution $\Delta^{\prime}=\left(\Sigma_{j}^{\prime}\right)_{j=1}^{m}$ is merged from $\Delta$ if $\Delta^{\prime}$ can be obtained from $\Delta=\left(\Sigma_{1}, \Sigma_{2}, \ldots, \Sigma_{n}\right)$ by merging sub-alphabets according to a proper partition of $[1, n]$, that is, if $\Delta^{\prime}$ satisfies the constraint that $\Sigma_{j}^{\prime}=\bigcup_{h \in \pi^{-1}(j)} \Sigma_{h}$ for each $j \in[1, m]$, where $\pi:[1, n] \mapsto[1, m]$ is a surjective function that induces a partition $\left\{\pi^{-1}(j) \mid j \in[1, m]\right\}$ of $[1, n]$ and we require $1<m<n$. Clearly, if $\Delta^{\prime}$ is merged from $\Delta$, then $\Delta \leq_{\Sigma} \Delta^{\prime}$ and $\left|\Delta^{\prime}\right|=m<n=|\Delta|$.

Remark 17. Note that the above surjective function $\pi$ cannot be arbitrarily specified, since $\Delta^{\prime}$ is by definition a distribution. For example, consider the alphabet $\Sigma=\{a, b, c, d, e\}$ and the distribution $\Delta=\left(\Sigma_{1}, \Sigma_{2}, \Sigma_{3}, \Sigma_{4}\right)$ of $\Sigma$, where $\Sigma_{1}=\{a, b\}$, $\Sigma_{2}=\{b, c\}, \Sigma_{3}=\{c, d\}$ and $\Sigma_{4}=\{d, e\}$. If we merge $\Sigma_{1}$ and $\Sigma_{3}$, then the resulting tuple $\left(\Sigma_{1} \cup \Sigma_{3}, \Sigma_{2}, \Sigma_{4}\right)$ is not a distribution, since $\Sigma_{2} \subseteq \Sigma_{1} \cup \Sigma_{3}$. Instead, we can merge $\Sigma_{1}$, $\Sigma_{2}$ and $\Sigma_{3}$ together, and then obtain the tuple $\left(\Sigma_{1} \cup \Sigma_{2} \cup\right.$ $\left.\Sigma_{3}, \Sigma_{4}\right)=(\{a, b, c, d\},\{d, e\})$, which is a distribution. To get rid of the above restriction on $\pi$, we only need to notice that $\left(\Sigma_{1} \cup \Sigma_{2} \cup \Sigma_{3}, \Sigma_{4}\right)$ is essentially equal to $\left(\Sigma_{1} \cup \Sigma_{3}, \Sigma_{4}\right)$, which can be obtained by keeping only those maximal sub-alphabets of $\left(\Sigma_{1} \cup \Sigma_{3}, \Sigma_{2}, \Sigma_{4}\right)$. Using this more general interpretation, which will also be used in the rest of this work, when we merge $\Sigma_{1}, \Sigma_{3}$ we indeed obtain the distribution $\left(\Sigma_{1} \cup \Sigma_{3}, \Sigma_{4}\right)$.

\footnotetext{
${ }^{13}$ Suppose, to the contrary, that $\left\{\Delta_{1}\right\}$ is a reduction of $\Delta$, then $\Delta \leq_{\Sigma} \Delta_{1}$ and $\Delta_{1} \leq_{\Sigma} \Delta$. This implies $\Delta=\Delta_{1}$. However, we require $\left|\Delta_{1}\right|<|\Delta|$ in Definition 6, which leads to a contradiction.
} 
Our next proposition shows that we only need to consider those distributions $\Delta_{i}$ 's that are merged from $\Delta$, when searching for reductions of any given distribution $\Delta$. Corollary 16 and Lemma 13 are needed for the proof.

Proposition 18. Suppose there exists a reduction $P$ of $\Delta$. Then there exists a reduction $Q$ of $\Delta$ such that the following two conditions hold: 1) each distribution in $Q$ is merged from $\Delta$; 2) the dimension of $Q$ is less than or equal to ${ }^{14}$ that of $P$.

Proof: Let $P=\left\{\Delta_{1}, \Delta_{2}, \ldots, \Delta_{l}\right\}$ be any given reduction of $\Delta$. Then, by Corollary $16, \Delta \leq_{\Sigma} \Delta_{i}$ for each $i \in[1, l]$. The idea is to show that for each $i \in[1, l]$ there exists a distribution $\Delta_{i}^{\prime}$ such that $\Delta \leq_{\Sigma} \Delta_{i}^{\prime} \leq_{\Sigma} \Delta_{i}, \Delta_{i}^{\prime}$ is merged from $\Delta$ and $\left|\Delta_{i}^{\prime}\right| \leq\left|\Delta_{i}\right|$. It then follows from Lemma 13 and the fact that $P$ is a reduction of $\Delta$ that $Q=\left\{\Delta_{1}^{\prime}, \Delta_{2}^{\prime}, \ldots, \Delta_{l}^{\prime}\right\}$ is a reduction of $\Delta$ that satisfies Conditions 1) and 2).

Let $\Delta=\left(\Sigma_{1}, \Sigma_{2}, \ldots, \Sigma_{n}\right), \Delta_{i}=\left(\Sigma_{i, 1}, \Sigma_{i, 2}, \ldots, \Sigma_{i, j_{i}}\right)$. Since $\Delta \leq_{\Sigma} \Delta_{i}$, there exists a function $f_{i}:[1, n] \mapsto\left[1, j_{i}\right]$ such that $\forall h \in[1, n], \Sigma_{h} \subseteq \Sigma_{i, f_{i}(h)}$. In the rest of the proof, we shall show that the distribution formed by those maximal sub-alphabets (in terms of set inclusion) in $\left\{\bigcup_{h \in f_{i}^{-1}(j)} \Sigma_{h} \mid\right.$ $\left.j \in f_{i}([1, n])\right\}$ can be chosen as $\Delta_{i}^{\prime}$.

Let $\Sigma_{i, j}^{\prime}=\bigcup_{h \in f_{i}-1(j)} \Sigma_{h}$ for each $j \in f_{i}([1, n])$. Suppose the tuple $\left(\Sigma_{i, j}^{\prime}\right)_{j \in f_{i}([1, n])}$ is indeed a distribution of $\Sigma$. Then, we have $\left(\Sigma_{i, j}^{\prime}\right)_{j \in f_{i}([1, n])} \leq_{\Sigma} \Delta_{i}$, since $\Sigma_{i, j}^{\prime} \subseteq \Sigma_{i, j}$ for each $j \in f_{i}([1, n])$. Also, it is clear that $\Delta \leq_{\Sigma}\left(\Sigma_{i, j}^{\prime}\right)_{j \in f_{i}([1, n])}$, since $\left(\Sigma_{i, j}^{\prime}\right)_{j \in f_{i}([1, n])}$ is merged from $\Delta$. Then, in this case, the tuple $\left(\Sigma_{i, j}^{\prime}\right)_{j \in f_{i}([1, n])}$ indeed can be chosen as $\Delta_{i}^{\prime}$. If the tuple $\left(\Sigma_{i, j}^{\prime}\right)_{j \in f_{i}([1, n])}$ is not a distribution, then the tuple formed by those maximal sub-alphabets in $\left\{\Sigma_{i, j}^{\prime} \mid j \in f_{i}([1, n])\right\}$ is a distribution that can be chosen as $\Delta_{i}^{\prime}$, essentially following the same reasoning.

We do not rule out the possibility that there are potentially some reductions of $\Delta$ whose distributions are not all merged from $\Delta$. However, Proposition 18 implies that for all practical purposes, searching among candidate reductions whose distributions are merged from $\Delta$ results in no loss of completeness. We remark that in the above proof of Proposition 18, $Q$ is linear time computable from $P$ and $\Delta$. Now, to finalize the search space for candidate reductions, we need to address the redundancy involved in a reduction and consider (a relaxation of) compact reductions.

Let $\Delta^{\prime}, \Delta^{\prime \prime}$ be any two distributions of $\Sigma . \Delta^{\prime}, \Delta^{\prime \prime}$ are said to be $\leq_{\Sigma}$-incomparable if $\Delta^{\prime} \not_{\Sigma} \Delta^{\prime \prime}$ and $\Delta^{\prime \prime} \not_{\Sigma} \Delta^{\prime}$. In a compact reduction, the distributions are guaranteed to be pairwise $\leq_{\Sigma}$-incomparable, which is a straightforward consequence of Lemma 13. However, a reduction whose distributions are $\leq_{\Sigma^{-}}$ incomparable is not necessarily compact. As an example, if Property $C$ ) holds, then $P=\left\{\left(\Sigma_{i}, \bigcup_{j \neq i} \Sigma_{j}\right) \mid i \in[1, n]\right\}$ is a reduction of $\Delta$ which is not compact, as we have discussed before. However, the distributions in $\left\{\left(\Sigma_{i}, \bigcup_{j \neq i} \Sigma_{j}\right) \mid i \in[1, n]\right\}$ are clearly $\leq_{\Sigma}$-incomparable. $\leq_{\Sigma}$-incomparability is a much simpler notion than compactness to verify. In order to show a reduction is not compact, we need to search for a proper subset that is a reduction; to show a reduction is compact, we need to prove that each proper subset is not a reduction. For each case, we shall also show a sound proof technique later. In this work, we shall mainly deal with reductions whose distributions

\footnotetext{
${ }^{14}$ We here use the usual partial ordering, i.e., $(l, k) \leq\left(l^{\prime}, k^{\prime}\right)$ if and only if $l \leq l^{\prime}$ and $k \leq k^{\prime}$.
}

are $\leq_{\Sigma}$-incomparable, as a means to reduce redundancy. The technique for establishing compactness could then be used to search for a compact reduction or verify compactness in a later stage.

Let $\mathcal{M}(\Delta)$ denote the set of distributions that are merged from $\Delta$. We only need to consider those reductions in which the distributions belong to $\mathcal{M}(\Delta)$ and are $\leq_{\Sigma}$-incomparable. Indeed, any set $P=\left\{\Delta_{1}, \Delta_{2}, \ldots, \Delta_{l}\right\} \in 2^{\mathcal{M}(\Delta)}-\{\varnothing\}$ of distributions could be modified to satisfy the $\leq_{\Sigma}$-incomparability condition by removing the non-minimal distributions (in terms of the partial ordering $\leq_{\Sigma}$ ) in the set, that is, by removing the distributions $\Delta_{i}$ 's that satisfy $\exists j \in[1, l],\left(\Delta_{j} \leq_{\Sigma} \Delta_{i} \wedge j \neq i\right)$; and the resultant set is denoted by $\widehat{P}$. Clearly, $\widehat{P}$ is a reduction if $P$ is. The search space for candidate reductions of $\Delta$ is then

$$
\begin{gathered}
S_{\Delta}=\left\{P \in 2^{\mathcal{M}(\Delta)}-\{\varnothing\} \mid \forall \Delta^{\prime}, \Delta^{\prime \prime} \in P,\left(\Delta^{\prime} \neq \Delta^{\prime \prime} \Longrightarrow\right.\right. \\
\left.\left.\Delta^{\prime} \not \Sigma_{\Sigma} \Delta^{\prime \prime}\right)\right\}
\end{gathered}
$$

In order to investigate the existence problem for reductions, we need to examine within $S_{\Delta}$ an order-theoretic structure of the set of reductions of $\Delta$. We shall first define a relation $\leq_{\Delta}$ on $S_{\Delta}$ (lifting the partial ordering $\leq_{\Sigma}$ ) as follows.

Definition 19. Given any $P=\left\{\Delta_{1}, \Delta_{2}, \ldots, \Delta_{l}\right\}$ and $Q=$ $\left\{\Delta_{1}^{\prime}, \Delta_{2}^{\prime}, \ldots, \Delta_{n}^{\prime}\right\}$ of $S_{\Delta}$, we define $P \leq_{\Delta} Q$ if $\forall j \in$ $[1, n], \exists i \in[1, l], \Delta_{i} \leq_{\Sigma} \Delta_{j}^{\prime}$.

It is not difficult to check that the relation $\leq_{\Delta}$ is a partial ordering. We assume that $\mathcal{M}(\Delta)$ is non-empty, and thus $S_{\Delta}$ is also non-empty. Then, the bottom element of $S_{\Delta}$ exists and is $\widehat{\mathcal{M}(\Delta)}$. A distribution $\Delta^{\prime}$ is said to be minimally merged from $\Delta$ if it can be obtained from $\Delta$ by merging two sub-alphabets $\Sigma_{i}$ and $\Sigma_{j}$, where $i \neq j$. Let $\perp(\Delta) \subseteq \mathcal{M}(\Delta)$ denote the set of minimally merged distributions from $\Delta$. We now show the following.

Proposition 20. $\widehat{\mathcal{M}(\Delta)}=\widehat{\perp(\Delta)}$.

Proof: $\mathcal{M}(\Delta)=\perp(\Delta) \cup(\mathcal{M}(\Delta)-\perp(\Delta))$. Each distribution $\Delta^{\prime}$ in $\mathcal{M}(\Delta)-\perp(\Delta)$, if it exists, is not minimally merged from $\Delta$. Thus, there exists a minimally merged distribution $\Delta^{\prime \prime} \in \perp(\Delta)$ such that $\Delta^{\prime \prime} \leq_{\Sigma} \Delta^{\prime}$. Thus, each distribution $\Delta^{\prime}$ in $\mathcal{M}(\Delta)-\perp(\Delta)$ is non-minimal in $\mathcal{M}(\Delta)$ and is removed in $\widehat{\mathcal{M}(\Delta)}$. Thus, we have $\widehat{\mathcal{M}(\Delta)}=\widehat{\perp(\Delta)}$.

That is, $\widehat{\perp(\Delta)}$ is the bottom element of $S_{\Delta}$. The following lemma states that the set of reductions of any given distribution $\Delta$ within $S_{\Delta}$ is indeed a downward closed set with respect to the partial ordering $\leq_{\Delta}$.

Lemma 21. Let $P, Q$ be any two elements of $S_{\Delta}$. If $P \leq_{\Delta} Q$ and $Q$ is a reduction of $\Delta$, then $P$ is also a reduction of $\Delta$.

Proof: Let $P=\left\{\Delta_{1}, \Delta_{2}, \ldots, \Delta_{l}\right\}$. We only need to show that given any language $L \subseteq \Sigma^{*}$, if $L$ is decomposable with respect to $\Delta_{i}$ for each $i \in[1, l]$, then $L$ is decomposable with respect to $\Delta$ (the other direction follows from Lemma 13). Let $Q=\left\{\Delta_{1}^{\prime}, \Delta_{2}^{\prime}, \ldots, \Delta_{n}^{\prime}\right\}$. For each $j \in[1, n]$, there exists $i \in[1, l]$, such that $\Delta_{i} \leq_{\Sigma} \Delta_{j}^{\prime}$, since $P \leq_{\Delta} Q$.

Suppose $L$ is decomposable with respect to $\Delta_{i}$ for $i \in[1, l]$. Then it follows that $L$ is decomposable with respect to $\Delta_{j}^{\prime}$ for each $j \in[1, n]$. Since $Q=\left\{\Delta_{1}^{\prime}, \Delta_{2}^{\prime}, \ldots, \Delta_{n}^{\prime}\right\}$ is a reduction of $\Delta$, we conclude that $L$ is decomposable with respect to $\Delta$ and thus $P$ is also a reduction of $\Delta$. 
We immediately obtain the next result, by using Lemma 21 and the fact that $\overline{\perp(\Delta)}$ is the bottom element of $S_{\Delta}$, i.e., the search space of candidate reductions.

Theorem 22. Let $\Delta$ be any distribution of $\Sigma$. Then $\Delta$ has a reduction if and only if $\overline{\perp(\Delta)}$ is a reduction of $\Delta$.

Thus, to determine the existence of a reduction for any given distribution $\Delta$ of size $n$ (Technical Problem 2), it suffices to check whether the set $\widehat{\perp(\Delta)}$ of distributions of dimension ${ }^{15}$ at most $(n(n-1) / 2, n-1)$ is a reduction. In general, however, there is still much redundancy involved in $\widehat{\perp(\Delta)}$, i.e., $\overline{\perp(\Delta)}$ is not compact. If $\Delta$ indeed has a reduction, then there is often a small subset of $\widehat{\perp(\Delta)}$ which is also a reduction of $\Delta$.

Example 23. For the distribution $\Delta$ discussed in Remark 17, the distribution $\Delta^{\prime}=(\{a, b, c, d\},\{d, e\})$ is minimally merged from $\Delta$, since it is obtained from $\Delta$ by merging $\Sigma_{1}$ and $\Sigma_{3}$. Let us now consider the distribution $\Delta$ in Example 9. We have $\perp(\Delta)=\left\{\Delta_{34}, \Delta_{12}, \Delta_{13}, \Delta_{14}, \Delta_{23}, \Delta_{24}\right\}$, where

$$
\left\{\begin{array}{l}
\Delta_{34}=(\{a, b\},\{b, c\},\{d, e, f\}) \\
\Delta_{12}=(\{d, e\},\{e, f\},\{a, b, c\}) \\
\Delta_{13}=(\{a, b, d, e\},\{b, c\},\{e, f\}) \\
\Delta_{14}=(\{a, b, e, f\},\{b, c\},\{d, e\}) \\
\Delta_{23}=(\{b, c, d, e\},\{a, b\},\{e, f\}) \\
\Delta_{24}=(\{b, c, e, f\},\{a, b\},\{d, e\})
\end{array}\right.
$$

Here $\Delta_{i j}$ denotes the distribution that is obtained from $\Delta$ by merging $\Sigma_{i}$ and $\Sigma_{j}$. In this example, distributions in $\perp(\Delta)$ are $\leq_{\Sigma}$-incomparable. Theorem 22 implies that the distribution $\Delta$ in Example 9 has a reduction if and only if $\overline{\perp(\Delta)}=\perp(\Delta)$ shown above is a reduction. From Example 9, we know the subset $\left\{\Delta_{34}, \Delta_{12}\right\}$, which exactly corresponds to $\left\{\Delta_{1}, \Delta_{2}\right\}$ in Example 9, of $\overline{\perp(\Delta)}$ is already a reduction of $\Delta$. Thus, $\perp(\Delta)$ is a reduction that is not compact.

The next lemma provides another necessary condition for a set of distributions to be a reduction of $\Delta$, which may be used to show that certain sets of distributions cannot be reductions of $\Delta$. This can be used to show that a distribution cannot have any reduction, by proving $\perp(\Delta)$ is not a reduction of $\Delta$, and a given reduction is compact, by proving that any proper subset of that reduction is not a reduction of $\Delta$ (Technical Problem 3).

Lemma 24. $\left\{\Delta_{1}, \Delta_{2}, \ldots, \Delta_{l}\right\}$ is a reduction of $\Delta$ only if $I_{\Delta}=\bigcup_{i \in[1, l]} I_{\Delta_{i}}$. Dually, $\left\{\Delta_{1}, \Delta_{2}, \ldots, \Delta_{l}\right\}$ is a reduction of $\Delta$ only if $D_{\Delta}=\bigcap_{i \in[1, l]} D_{\Delta_{i}}$.

Lemma 24 can be further strengthened and generalized. Let $\Delta=\left(\Sigma_{1}, \Sigma_{2}, \ldots, \Sigma_{n}\right)$ be any distribution of $\Sigma$. Let $\Sigma^{\prime}$ be any non-empty subset of $\Sigma$ such that $\left|\Sigma^{\prime}\right| \geq 2$. We define $\Sigma^{\prime} \sqsubseteq \Delta$ if $\exists i \in[1, n], \Sigma^{\prime} \subseteq \Sigma_{i}$. Intuitively, we use $\Sigma^{\prime}$ to capture the mutual dependence of at least two events. We have the next lemma, whose proof relies on Lemma 15 and Corollary 16.

Lemma 25. Let $\Delta$ be any distribution of $\Sigma$ and let $\Delta_{i}$ be any distribution of $\Sigma$ for each $i \in[1, l] .\left\{\Delta_{1}, \Delta_{2}, \ldots, \Delta_{l}\right\}$ is a reduction of $\Delta$ only if for each subset $\Sigma^{\prime}$ of $\Sigma$ of cardinality at least $2,\left(\forall i \in[1, l], \Sigma^{\prime} \sqsubseteq \Delta_{i}\right) \Longleftrightarrow \Sigma^{\prime} \sqsubseteq \Delta$.

\footnotetext{
${ }^{15}$ Here we have used a general definition of dimension in a natural way, for sets of distributions.
}

Proof: Suppose the set $\left\{\Delta_{1}, \Delta_{2}, \ldots, \Delta_{l}\right\}$ is a reduction of $\Delta$. Now, let $\Sigma^{\prime}$ be any subset of $\Sigma$ with $\left|\Sigma^{\prime}\right| \geq 2$. Clearly, we have $\Sigma^{\prime} \sqsubseteq \Delta \Longrightarrow\left(\forall i \in[1, l], \Sigma^{\prime} \sqsubseteq \Delta_{i}\right)$, since $\Delta \leq_{\Sigma} \Delta_{i}$ for each $i \in[1, l]$, by Corollary 16. Suppose $\forall i \in[1, l], \Sigma^{\prime} \sqsubseteq \Delta_{i}$. Each language $L \subseteq \Sigma^{\prime *}$, when viewed as a language over $\Sigma$, is decomposable with respect to each $\Delta_{i}$, where $i \in[1, l]$. This implies that every language $L \subseteq \Sigma^{\prime *}$ over $\Sigma$ is decomposable with respect to $\Delta$, since $\left\{\Delta_{1}, \Delta_{2}, \ldots, \Delta_{l}\right\}$ is by supposition a reduction of $\Delta$. But this implies $\Sigma^{\prime} \sqsubseteq \Delta$, since otherwise there would exist a language $L \subseteq \Sigma^{*}$ which, when viewed as a language over $\Sigma$, is not decomposable with respect to $\Delta$ (by the proof of Lemma 15), resulting in a contradiction.

We have the following, by Theorem 22 and Lemma 25 .

Corollary 26. Let $\widehat{\perp(\Delta)}=\left\{\Delta_{1}, \Delta_{2}, \ldots, \Delta_{l}\right\}$. If there exists a subset $\Sigma^{\prime}$ of $\Sigma$ of cardinality at least 2 such that $(\forall i \in$ $\left.[1, l], \Sigma^{\prime} \sqsubseteq \Delta_{i}\right) \wedge \Sigma^{\prime} \nsubseteq \Delta$, then $\Delta$ does not have a reduction.

Example 27. Consider the alphabet $\Sigma=\{a, b, c, d, e, f\}$ and the distribution $\Delta=\left(\Sigma_{1}, \Sigma_{2}, \Sigma_{3}\right)$ of $\Sigma$, where $\Sigma_{1}=\{a, b, c\}$, $\Sigma_{2}=\{c, d, e\}, \Sigma_{3}=\{e, f, a\} . \overline{\perp(\Delta)}=\left\{\Delta_{1}, \Delta_{2}, \Delta_{3}\right\}$ is not a reduction of $\Delta$, where

$$
\left\{\begin{array}{l}
\Delta_{1}=(\{a, b, c, d, e\},\{e, f, a\}) \\
\Delta_{2}=(\{a, b, c, e, f\},\{c, d, e\}) \\
\Delta_{3}=(\{a, c, d, e, f\},\{a, b, c\})
\end{array}\right.
$$

since $\{a, c, e\} \sqsubseteq \Delta_{i}$ for each $i \in[1,3]$ but $\{a, c, e\} \nsubseteq \Delta$. Thus, according to Corollary 26, $\Delta$ cannot have any reduction. In this example, Lemma 24 is not strong enough to conclude $\Delta$ does not have a reduction, since $I_{\Delta}=\bigcup_{i=1}^{3} I_{\Delta_{i}}$.

Lemma 25 provides a sound technique for proving a reduction to be compact. In fact, to prove a reduction is compact, we only need to consider all the proper subsets and prove they are not reductions using Lemma 25 . This is now illustrated by the following example.

Example 28. Consider the reduction $\left\{\Delta_{1}^{\prime}, \Delta_{2}^{\prime}, \Delta_{3}^{\prime}\right\}$ of distribution $\Delta=\left(\Sigma_{1}, \Sigma_{2}, \Sigma_{3}, \Sigma_{4}\right)$ of $\Sigma=\{a, b, c, d, e, f\}$ shown in Example 9, where $\Sigma_{1}=\{a, b\}, \Sigma_{2}=\{b, c\}, \Sigma_{3}=\{d, e\}$, $\Sigma_{4}=\{e, f\}$ and

$$
\left\{\begin{aligned}
\Delta_{1}^{\prime} & =(\{a, b\},\{b, c, d, e, f\}) \\
\Delta_{2}^{\prime} & =(\{a, b, c\},\{d, e, f\}) \\
\Delta_{3}^{\prime} & =(\{d, e\},\{a, b, c, e, f\})
\end{aligned}\right.
$$

The reduction $\left\{\Delta_{1}^{\prime}, \Delta_{2}^{\prime}, \Delta_{3}^{\prime}\right\}$ is compact, since $\{d, e, f\}$ refutes $\left\{\Delta_{1}^{\prime}, \Delta_{2}^{\prime}\right\}$ as a reduction of $\Delta,\{b, e, f\}$ refutes $\left\{\Delta_{1}^{\prime}, \Delta_{3}^{\prime}\right\}$ and $\{a, b, c\}$ refutes $\left\{\Delta_{2}^{\prime}, \Delta_{3}^{\prime}\right\}$.

Lemma 25 could be used to further reduce the search space $S_{\Delta}$ in a straightforward manner and the resultant search space of candidate reductions is denoted by $S_{\Delta}^{\prime}$. In order to tackle the problem of reduction computation, it is critical to solve the problem of reduction verification. Unfortunately, the decidability status of the problem of reduction verification still remains unknown. The next proposition shows that the problem is corecursively enumerable and will render a simplification of the notion of reduction of distributions (see Corollary 30 and also Remark 7). We shall note that each candidate reduction in $S_{\Delta}^{\prime}$ satisfies the condition of Lemma 24, i.e., $I_{\Delta}=\bigcup_{i \in[1, l]} I_{\Delta_{i}}$.

Proposition 29. If a set $\left\{\Delta_{1}, \Delta_{2}, \ldots, \Delta_{l}\right\} \in S_{\Delta}^{\prime}$ of distributions of $\Sigma$ is not a reduction of $\Delta$, then there must exist a 
finite language $L$ over $\Sigma$ that is decomposable with respect to each $\Delta_{i}$, but not decomposable with respect to $\Delta$.

Proof: Suppose $L \subseteq \Sigma^{*}$ is a language that is decomposable with respect to each $\Delta_{i}$, where $i \in[1, l]$, but not decomposable with respect to $\Delta$. The existence of such an $L$ is guaranteed since $\left\{\Delta_{1}, \Delta_{2}, \ldots, \Delta_{l}\right\} \in S_{\Delta}^{\prime}$ is not a reduction of $\Delta$. We now show that there exists a finite sublanguage $L_{f}$ of $L$ that is decomposable with respect to each $\Delta_{i}$, but not decomposable with respect to $\Delta$.

We first observe that since $L$ is decomposable with respect to each $\Delta_{i}$, then by Lemma $2, L$ is trace-closed with respect to each $I_{\Delta_{i}}$. By Lemma 5, we conclude that $L$ is trace-closed with respect to $I_{\Delta}$, i.e., $L=[L]_{I_{\Delta}}$, since $I_{\Delta}=\bigcup_{i \in[1, l]} I_{\Delta_{i}}$. Let $\Delta=\left(\Sigma_{1}, \Sigma_{2}, \ldots, \Sigma_{n}\right)$. It is always possible to choose $n$ strings $s_{1}, s_{2}, \ldots, s_{n} \in L$ such that $\|_{i=1}^{n} P_{i}\left(s_{i}\right) \cap L^{c} \neq \varnothing$, since $L$ is not decomposable with respect to $\Delta$, i.e., $L \neq \|_{i=1}^{n} P_{i}(L)$. Let $L^{\prime}=\left\{s_{1}, s_{2}, \ldots, s_{n}\right\} \subseteq L$, which is by definition a finite language over $\Sigma$. We note that it is always possible to compute the infimal decomposable superlanguage $L_{f}$ of $L^{\prime}$ with respect to the distributions $\Delta_{1}, \Delta_{2}, \ldots, \Delta_{l}$, the existence of which is ensured since the class of decomposable superlanguages of any given language is closed under arbitrary intersection, and the language $\Sigma^{*}$ is decomposable with respect to the distributions $\Delta_{1}, \Delta_{2}, \ldots, \Delta_{l}$.

Indeed, let $\Sigma$ be enumerated as $\left\{\sigma_{1}, \sigma_{2}, \ldots, \sigma_{|\Sigma|}\right\}$ and we define $\Delta^{\prime}=\left(\Sigma_{1}, \Sigma_{2}, \ldots, \Sigma_{|\Sigma|}\right)$, where $\Sigma_{i}=\left\{\sigma_{i}\right\}$. It is clear that $L_{f}$ defined above is a finite language, since $L_{f} \subseteq L^{\prime \Delta^{\prime}}$ (note that $\Delta^{\prime} \leq_{\Sigma} \Delta_{i}$ for each $i \in[1, l]$ ) and $L^{\prime \Delta^{\prime}} \subseteq \Sigma^{*}$ is a finite language. Now, since $L$ is decomposable with respect to $\Delta_{1}, \Delta_{2}, \ldots, \Delta_{l}$ and $L^{\prime} \subseteq L$, we conclude that $L_{f} \subseteq L$. We shall now show that $L_{f}$ is not decomposable with respect to $\Delta$. Indeed, we have $\left\|_{i=1}^{n} P_{i}\left(s_{i}\right) \subseteq\right\|_{i=1}^{n} P_{i}\left(L^{\prime}\right) \subseteq \|_{i=1}^{n} P_{i}\left(L_{f}\right)$. Thus, $\|_{i=1}^{n} P_{i}\left(L_{f}\right) \cap L^{c} \neq \varnothing$. However, $L_{f} \cap L^{c}=\varnothing$ since $L_{f} \subseteq L$. We conclude that $L_{f} \neq \|_{i=1}^{n} P_{i}\left(L_{f}\right)$, that is, $L_{f}$ is not decomposable with respect to $\Delta$.

It is of significant interest to know whether it is possible to upper bound (in some way) the length of the longest strings in $L^{\prime}$ in terms of the sizes of the inputs $\Delta_{1}, \Delta_{2}, \ldots, \Delta_{l}$ and $\Delta$. If this is possible, then the proof of Proposition 29 will imply that the problem of reduction verification is decidable, since an upper bound on the length of the longest strings in $L_{f}$ can be computed from an upper bound on the length of the longest strings in $L^{\prime}$. We can use Proposition 29 to reformulate the definition of reduction of distributions.

Corollary 30. A set $\left\{\Delta_{1}, \Delta_{2}, \ldots, \Delta_{l}\right\}$ of distributions is a reduction of $\Delta$ if the following conditions hold: 1) an arbitrary finite language $L \subseteq \Sigma^{*}$ is decomposable with respect to $\Delta_{i}$ for each $i \in[1, l]$ if and only if it is decomposable with respect to $\Delta$; 2) for each $i \in[1, l],\left|\Delta_{i}\right|<|\Delta|$.

A distribution could have more than one reduction, but a set of distributions can only be the reduction of at most one distribution (see Technical Problem 5). This is a straightforward consequence of Lemma 15 and is stated as follows.

Corollary 31. Let $\Delta, \Delta^{\prime}$ be any two distributions. If a set of distributions is a reduction of both $\Delta$ and $\Delta^{\prime}$. Then, $\Delta=\Delta^{\prime}$. Equivalently, if a set $P$ of distributions is a reduction of $\Delta^{\prime}$ and $\Delta \neq \Delta^{\prime}$, then $P$ cannot be a reduction of $\Delta$.

\section{STRUCTURES OF Distributions AND Substitution-BASEd Proof}

In this section, we study two different structures of distributions: 1) the existence of an eavesdropping sub-alphabet; 2) the existence of multiple connected components in the "nondisjointness" graph induced by the distribution. It turns out that the property of having an eavesdropping sub-alphabet is much weaker ${ }^{16}$ than Property $C$ ), yet exactly the same reduction can be obtained; and two different reductions are shown for distributions whose induced non-disjointness graphs have multiple connected components. These two special structures are used to convince the readers about the abundance of the structures (of distributions) as well as the usefulness of reduction-based approach. A substitution-based proof technique for verifying reductions of distributions will be illustrated on these distributions. And the proof idea can be easily adapted for automatic verification of reductions of distributions.

\section{A. Eavesdropping}

Any alphabet $\Sigma_{0} \subseteq \Sigma$ is said to eavesdrop distribution $\Delta=$ $\left(\Sigma_{1}, \Sigma_{2}, \ldots, \Sigma_{n}\right)$ of $\Sigma$ if $\bigcup_{i \neq j}\left(\Sigma_{i} \cap \Sigma_{j}\right) \subseteq \Sigma_{0}$. We note that if $\Sigma_{k}$, where $k \in[1, n]$, eavesdrops $\Delta$, then $\Delta_{i}=\left(\Sigma_{i}, \bigcup_{j \neq i} \Sigma_{j}\right)$ is indeed a distribution ${ }^{17}$ for each $i \neq k$. The following result generalizes Theorem 8 of [3]. For each set $I \subseteq[1, n], P_{I}$ : $\Sigma^{*} \mapsto\left(\bigcup_{i \in I} \Sigma_{i}\right)^{*}$ is used to denote the natural projection.

Proposition 32. If $\Sigma_{k}$ eavesdrops $\Delta=\left(\Sigma_{1}, \Sigma_{2}, \ldots, \Sigma_{n}\right)$, then the set $\left\{\Delta_{i} \mid i \neq k\right\}$ of distributions is an $(n-1,2)$-reduction of $\Delta$, where $\Delta_{i}=\left(\Sigma_{i}, \bigcup_{j \neq i} \Sigma_{j}\right)$.

Proof: Since each distribution $\Delta_{i}$ is merged from $\Delta$, we only need to show that the decomposability of $L$ with respect to these $\Delta_{i}$ 's implies the decomposability of $L$ with respect to $\Delta$. Suppose $L$ is decomposable with respect to $\Delta_{i}$ 's, where $i \neq k$, and assume, without loss of generality, that $k=n$. Then we have the following.

$$
\left\{\begin{array}{c}
L=P_{1}(L) \| P_{[1, n]-\{1\}}(L) \quad \ldots(1) \\
L=P_{2}(L) \| P_{[1, n]-\{2\}}(L) \quad \ldots(2) \\
L=P_{3}(L) \| P_{[1, n]-\{3\}}(L) \quad \ldots(3) \\
\ldots \\
L=P_{n-1}(L) \| P_{[1, n]-\{n-1\}}(L) \quad \ldots(\mathrm{n}-1)
\end{array}\right.
$$

By substituting Equation (2) into Equation (1), we have, by Lemma 3 , the following

$$
\begin{gathered}
L=P_{1}(L) \| P_{[1, n]-\{1\}}\left(P_{2}(L) \| P_{[1, n]-\{2\}}(L)\right)= \\
P_{1}(L)\left\|P_{2}(L)\right\| P_{[1, n]-\{1,2\}}(L) \quad \ldots(1[2])
\end{gathered}
$$

since $\Sigma_{2} \subseteq \bigcup_{j \neq 1} \Sigma_{j}$ and

$$
\begin{gathered}
\left(\bigcup_{j \neq 1} \Sigma_{j}\right) \cap\left(\bigcup_{j \neq 2} \Sigma_{j}\right)=\bigcup_{j \in[1, n]-\{1,2\}} \Sigma_{j} \cup\left(\Sigma_{1} \cap \Sigma_{2}\right)= \\
\bigcup_{j \in[1, n]-\{1,2\}} \Sigma_{j} \text {, since } \Sigma_{1} \cap \Sigma_{2} \subseteq \Sigma_{n}
\end{gathered}
$$

By substituting Equation (3) into Equation (1[2]) in a similar way, we obtain, by Lemma 3 , the following

$$
L=P_{1}(L)\left\|P_{2}(L)\right\| P_{3}(L) \| P_{[1, n]-\{1,2,3\}}(L)
$$

By successive substitutions of the remaining equations in a similar way, we eventually obtain that

$$
L=\left\|_{i=1}^{n-1} P_{i}(L)\right\| P_{[1, n]-\{1,2, \ldots, n-1\}}(L)=\|_{i=1}^{n} P_{i}(L)
$$

That is, $L$ is decomposable with respect to $\Delta$.

\footnotetext{
${ }^{16}$ Any distribution $\Delta$ that satisfies Property $C$ ) has an eavesdropping subalphabet and in fact, any sub-alphabet is eavesdropping if Property $C$ ) holds

${ }^{17}$ Otherwise, it can be shown that $\Sigma_{i} \subseteq\left(\bigcup_{j \neq i} \Sigma_{j}\right) \cap \Sigma_{i} \subseteq \Sigma_{k}$, which contradicts the fact that $\Delta$ is a distribution.
} 
Example 33. Consider the alphabet $\Sigma=\{a, b, c, d, e\}$ and the distribution $\Delta=(\{a, c\},\{b, d\},\{a, e\},\{a, b\})$ of $\Sigma$. Clearly, the sub-alphabet $\{a, b\}$ eavesdrops $\Delta$. Thus, $\left\{\Delta_{1}, \Delta_{2}, \Delta_{3}\right\}$ is a reduction of $\Delta$ according to Proposition 32, where

$$
\left\{\begin{array}{l}
\Delta_{1}=(\{a, c\},\{a, b, d, e\}) \\
\Delta_{2}=(\{b, d\},\{a, b, c, e\}) \\
\Delta_{3}=(\{a, e\},\{a, b, c, d\})
\end{array}\right.
$$

Note that Property C) is not satisfied by the distribution in this example.

\section{B. Multiple Connected Components}

Given any distribution $\Delta=\left(\Sigma_{1}, \Sigma_{2}, \ldots, \Sigma_{n}\right)$, it is possible to define an undirected graph $G(\Delta)=(V(\Delta), E(\Delta))$, i.e., the non-disjointness graph induced by $\Delta$, as follows. Let $V(\Delta)=\left\{v_{1}, v_{2}, \ldots, v_{n}\right\}$ and we can define a bijective map $f: V(\Delta) \mapsto\left\{\Sigma_{1}, \Sigma_{2}, \ldots, \Sigma_{n}\right\}$ such that $f\left(v_{i}\right)=\Sigma_{i}$. Let $E(\Delta)=\left\{\left(v, v^{\prime}\right) \in V(\Delta) \times V(\Delta) \mid v \neq v^{\prime} \wedge f(v) \cap f\left(v^{\prime}\right) \neq\right.$ $\varnothing\}$. Let $\left\{C_{1}, C_{2}, \ldots, C_{k}\right\}$ be the set of connected components of $G(\Delta)$, where $C_{i}$ will also be used to denote the vertices of the $i$-th connected component. Clearly, $C_{i} \cap C_{j}=\varnothing$, for $i \neq j$, and $\bigcup_{i=1}^{k} C_{i}=V(\Delta)$, i.e., the collection $\left\{C_{1}, C_{2}, \ldots, C_{k}\right\}$ is a partition of $V(\Delta)$. For any $V^{\prime} \subseteq V(\Delta)$, let $I\left(V^{\prime}\right)=$ $\left\{j \in[1, n] \mid v_{j} \in V^{\prime}\right\}$ denote the index set for the vertices of $V^{\prime}$. Let $d(\Delta)=\max _{i \in[1, k]}\left|C_{i}\right|$. We are now ready to state Proposition 34. We note that all the tuples involved are indeed distributions.

Proposition 34. Let $\left\{C_{1}, C_{2}, \ldots, C_{k}\right\}$ be the set of connected components of $G(\Delta)$. If $k \geq 2$, then the set $\left\{\Delta_{i} \mid i \in[1, k]\right\}$ of distributions is a $(k, d(\Delta)+1)$-reduction of $\Delta$, where $\Delta_{i}=$ $\left(\left(\Sigma_{j}\right)_{j \in I\left(C_{i}\right)}, \bigcup_{j \notin I\left(C_{i}\right)} \Sigma_{j}\right)$. If, in addition, $\left|C_{h}\right|=1$, for some $h \in[1, k]$, then $\left\{\Delta_{i} \mid i \in[1, k], i \neq h\right\}$ is a $(k-1, d(\Delta)+1)$ reduction of $\Delta$.

Proof: Since each distribution $\Delta_{i}$ is merged from $\Delta$, we only need to show that the decomposability of $L$ with respect to these $\Delta_{i}$ 's implies the decomposability of $L$ with respect to $\Delta$. Suppose $L$ is decomposable with respect to $\Delta_{i}$ 's. Then we have the following.

$$
\left\{\begin{array}{c}
L=\left\|_{j \in I\left(C_{1}\right)} P_{j}(L)\right\| P_{I\left(V-C_{1}\right)}(L) \quad \ldots(1) \\
L=\left\|_{j \in I\left(C_{2}\right)} P_{j}(L)\right\| P_{I\left(V-C_{2}\right)}(L) \quad \ldots(2) \\
\ldots \\
L=\left\|_{j \in I\left(C_{k-1}\right)} P_{j}(L)\right\| P_{I\left(V-C_{k-1}\right)}(L) \ldots(\mathrm{k}-1) \\
L=\left\|_{j \in I\left(C_{k}\right)} P_{j}(L)\right\| P_{I\left(V-C_{k}\right)}(L) \quad \ldots(\mathrm{k})
\end{array}\right.
$$

By substituting Equation (2) into Equation (1), we have, by Lemma 3, the following

$$
\begin{gathered}
L= \\
\left\|_{j \in I\left(C_{1}\right)} P_{j}(L)\right\| P_{I\left(V-C_{1}\right)}\left(\left\|_{j \in I\left(C_{2}\right)} P_{j}(L)\right\| P_{I\left(V-C_{2}\right)}(L)\right) \\
\left(\|_{j \in I\left(C_{1}\right)} P_{j}(L)\right)\left\|\left(\|_{j \in I\left(C_{2}\right)} P_{j}(L)\right)\right\| P_{I\left(V-\left(C_{1} \cup C_{2}\right)\right)}(L) \\
\ldots(1[2])
\end{gathered}
$$

since $I\left(C_{2}\right) \subseteq I\left(V-C_{1}\right)$ and $\forall j \in I\left(C_{1}\right), \forall l \in I\left(C_{2}\right), \Sigma_{j} \cap$ $\Sigma_{l}=\varnothing$. We then continue the above analysis by successive substitutions of the remaining equations. Upon the substitution of Equation $(k-1)$, we obtain the equality

$$
L=\left(\|_{j \in I\left(C_{1}\right)} P_{j}(L)\right)\|\ldots\|\left(\|_{j \in I\left(C_{k-1}\right)} P_{j}(L)\right) \| P_{I\left(C_{k}\right)}(L)
$$

Upon the final substitution of Equation $(k)$, we have

$$
L=\left\|_{i=1}^{k}\left(\|_{j \in I\left(C_{i}\right)} P_{j}(L)\right)=\right\|_{j=1}^{n} P_{j}(L)
$$

That is, $L$ is decomposable with respect to $\Delta$. If, in addition, $\left|C_{h}\right|=1$, for some $h \in[1, k]$, and assume, without loss of generality, that $\left|C_{k}\right|=1$, then upon the $(k-1)$ th substitution the equality $L=\|_{j=1}^{n} P_{j}(L)$ has been obtained.

Remark 35. In Proposition 34, each $\Delta_{i}$ is obtained from $\Delta$ by merging those sub-alphabets whose corresponding vertices are in connected components $C_{j}$ 's, where $j \neq i$.

Example 36. Consider the distribution $\Delta=\left(\Sigma_{1}, \Sigma_{2}, \Sigma_{3}, \Sigma_{4}\right)$ of $\Sigma=\{a, b, c, d, e, f\}$, where $\Sigma_{1}=\{a, b\}, \Sigma_{2}=\{b, c\}$, $\Sigma_{3}=\{d, e\}$ and $\Sigma_{4}=\{e, f\}$. There are two connected components $C_{1}=\left\{v_{1}, v_{2}\right\}$ and $C_{2}=\left\{v_{3}, v_{4}\right\}$. Thus, $I\left(C_{1}\right)=$ $\{1,2\}$ and $I\left(C_{2}\right)=\{3,4\}$. According to Proposition 34, the set $\left\{\Delta_{1}, \Delta_{2}\right\}$ of distributions, where $\Delta_{1}=\left(\Sigma_{1}, \Sigma_{2}, \Sigma_{3} \cup \Sigma_{4}\right)$ and $\Delta_{2}=\left(\Sigma_{3}, \Sigma_{4}, \Sigma_{1} \cup \Sigma_{2}\right)$, is a $(2,3)$-reduction of $\Delta$. This indeed corresponds to the reduction $\left\{\Delta_{1}, \Delta_{2}\right\}$ in Example 9.

An alternative reduction for distributions of the same structure is shown in Proposition 37. For convenience, we arbitrarily organize $I\left(C_{1}\right), I\left(C_{2}\right), \ldots, I\left(C_{k}\right)$ into a $k \times d(\Delta)$ matrix $M_{\Delta}$ such that, for each $i \in[1, k]$, the $i$-th row corresponds to the elements of $I\left(C_{i}\right)$ followed by $d(\Delta)-\left|C_{i}\right| 0$ 's. Let $M_{\Delta}[j]$ denote the set of non-zero elements of $M_{\Delta}$ in the $j$-th column. We note that all the involved tuples in Proposition 37 are indeed distributions.

Proposition 37. Let $\left\{C_{1}, C_{2}, \ldots, C_{k}\right\}$ be the set of connected components of $G(\Delta)$. If $k \geq 2$, then the set $\left\{\Delta_{1}, \Delta_{2}\right\}$ of distributions is a $(2, \max (k, d(\Delta)))$-reduction of $\Delta$, where $\Delta_{1}=\left(\bigcup_{l \in M_{\Delta}[j]} \Sigma_{l}\right)_{j=1}^{d(\Delta)}$ and $\Delta_{2}=\left(\bigcup_{j \in I\left(C_{i}\right)} \Sigma_{j}\right)_{i=1}^{k}$.

Proof: Since both $\Delta_{1}, \Delta_{2}$ are merged from $\Delta$, we only need to show that the decomposability of $L$ with respect to $\Delta_{1}, \Delta_{2}$ implies the decomposability of $L$ with respect to $\Delta$. Suppose $L$ is decomposable with respect to $\Delta_{1}, \Delta_{2}$. Then we have the following.

$$
\left\{\begin{array}{l}
L=\|_{j=1}^{d(\Delta)} P_{M_{\Delta}[j]}(L) \quad \ldots(1) \\
L=\|_{i=1}^{k} P_{I\left(C_{i}\right)}(L)
\end{array}\right.
$$

By substituting Equation (2) into Equation (1), we immediately obtain, by Lemma 3 , the desired result

$$
\begin{gathered}
L=\|_{j=1}^{d(\Delta)} P_{M_{\Delta}[j]}\left(\|_{i=1}^{k} P_{I\left(C_{i}\right)}(L)\right)= \\
\left\|_{j=1}^{d(\Delta)}\right\|_{i=1}^{k} P_{I\left(C_{i}\right) \cap M_{\Delta}[j]}(L)=\|_{i=1}^{n} P_{i}(L)
\end{gathered}
$$

since $\left(\bigcup_{l \in I\left(C_{i}\right)} \Sigma_{l}\right) \cap \bigcup_{l \in I\left(C_{j}\right)} \Sigma_{l}=\emptyset$ for $i \neq j$ and the set of non-zero elements of $M_{\Delta}$ is exactly equal to $[1, n]$.

Remark 38. In Proposition 37, $\Delta_{1}$ (respectively, $\Delta_{2}$ ) is obtained from $\Delta$ by merging those sub-alphabets whose indices are in the same column (respectively, row) of $M_{\Delta}$.

Example 39. The two reductions $\left\{\Delta_{1}^{\prime \prime}, \Delta_{2}^{\prime \prime}\right\}$ and $\left\{\Delta_{1}^{\prime \prime \prime}, \Delta_{2}^{\prime \prime \prime}\right\}$ shown in Example 9 can be obtained using Proposition 37. Let us now consider the alphabet $\Sigma=\{a, b, c, d, e, f, g\}$ and the distribution $\Delta=\left(\Sigma_{1}, \Sigma_{2}, \Sigma_{3}, \Sigma_{4}, \Sigma_{5}\right)$ of $\Sigma$, where $\Sigma_{1}=$ $\{a, b\}, \Sigma_{2}=\{b, c\}, \Sigma_{3}=\{c, d\}, \Sigma_{4}=\{e, f\}$ and $\Sigma_{5}=$ $\{f, g\}$. Clearly, there are two connected components $C_{1}=$ $\left\{v_{1}, v_{2}, v_{3}\right\}$ and $C_{2}=\left\{v_{4}, v_{5}\right\}$. Thus, $I\left(C_{1}\right)=\{1,2,3\}$ and $I\left(C_{2}\right)=\{4,5\}$. The matrix $M_{\Delta}$ is then shown below.

$$
\left(\begin{array}{lll}
1 & 2 & 3 \\
4 & 5 & 0
\end{array}\right)
$$

According to Proposition 37, the set $\left\{\Delta_{1}, \Delta_{2}\right\}$ of distributions, where $\Delta_{1}=\left(\Sigma_{1} \cup \Sigma_{4}, \Sigma_{2} \cup \Sigma_{5}, \Sigma_{3}\right)$ and $\Delta_{2}=\left(\Sigma_{1} \cup \Sigma_{2} \cup\right.$ $\left.\Sigma_{3}, \Sigma_{4} \cup \Sigma_{5}\right)$, is a (2,3)-reduction of $\Delta$. 
We now summarize the main idea behind the substitutionbased proof technique, by abstracting away the languages and focusing only on the operations performed on the distributions. For any distribution $\Delta=\left(\Sigma_{1}, \Sigma_{2}, \ldots, \Sigma_{n}\right)$ of $\Sigma$, let $A(\Delta)=$ $\bigcup_{i \neq j}\left(\Sigma_{i} \cap \Sigma_{j}\right)$ denote the set of shared events in $\Delta$. Let $\Delta^{\prime}=$ $\left(\Sigma_{1}^{\prime}, \Sigma_{2}^{\prime}, \ldots, \Sigma_{l}^{\prime}\right)$ be another distribution of $\Sigma . \Delta$ is said to be substitutable into the $i$-th sub-alphabet of $\Delta^{\prime}$, denoted $\Delta \rightarrow i$ $\Delta^{\prime}$, if $A(\Delta) \subseteq \Sigma_{i}^{\prime}$ holds. $\Delta$ is said to be substitutable into $\Delta^{\prime}$, denoted $\Delta \rightarrow \Delta^{\prime}$, if $\Delta \rightarrow_{i} \Delta^{\prime}$ for some $i \in[1, l]$. The result of substituting $\Delta$ into the $i$-th sub-alphabet of $\Delta^{\prime}$ is denoted by $\left(\Delta \vdash_{i} \Delta^{\prime}\right)$, which is defined to be the distribution obtained from

$$
\begin{gathered}
\left(\Sigma_{1}^{\prime}, \Sigma_{2}^{\prime}, \ldots, \Sigma_{i-1}^{\prime}, \Sigma_{i}^{\prime} \cap \Sigma_{1}, \Sigma_{i}^{\prime} \cap \Sigma_{2}, \ldots, \Sigma_{i}^{\prime} \cap\right. \\
\left.\Sigma_{n}, \Sigma_{i+1}^{\prime}, \ldots, \Sigma_{l}^{\prime}\right)
\end{gathered}
$$

by deleting those non-maximal components. And the result of substituting $\Delta$ into $\Delta^{\prime}$ is denoted by $\left(\Delta \vdash \Delta^{\prime}\right)$ and is defined to be the set $\left\{\left(\Delta \vdash_{i} \Delta^{\prime}\right) \mid \Delta \neg_{i} \Delta^{\prime}, i \in[1, l]\right\}$. Then, the idea of the above proof technique is to derive new distributions by using substitutions and maintain the set of currently available distributions. If $\Delta$ can be computed from $\left\{\Delta_{1}, \Delta_{2}, \ldots, \Delta_{n}\right\}$ in this way and also $\Delta \leq_{\Sigma} \Delta_{i}$ holds for each $i \in[1, n]$, then $\left\{\Delta_{1}, \Delta_{2}, \ldots, \Delta_{n}\right\}$ is a reduction of $\Delta$, using Lemma 3 and Lemma 13. Clearly, this process can be mechanized.

It is unclear whether the substitution-based proof technique is complete. The following example shows that the refutation technique based on Lemma 25 is not complete. In the unfortunate case when all previously developed techniques fail, any attempt to refute a reduction currently entails the search for a finite language counterexample (Proposition 29).

Example 40. Let $\left\{\Delta_{1}, \Delta_{2}\right\}$ be a set of distributions, where $\Delta_{1}=(\{a, b, c\},\{a, c, d\})$ and $\Delta_{2}=(\{b, c, d\},\{a, b, d\})$. Consider the distribution $\Delta=(\{a, b\},\{b, c\},\{c, d\},\{d, a\})$. Clearly, both $\Delta_{1}$ and $\Delta_{2}$ are merged from $\Delta$. Furthermore, the necessary condition in Lemma 25 is satisfied. However, neither is $\Delta_{1}$ substitutable into $\Delta_{2}$ nor is $\Delta_{2}$ substitutable into $\Delta_{1}$. We cannot validate the candidate reduction $\left\{\Delta_{1}, \Delta_{2}\right\}$ nor refute it, using the techniques developed so far. It is not difficult to check that the finite language

$\{a b b c d, a b c d d, a b b c d d, a b c d, d c b b a, d c b b a a, d c c b b a, d c c b b a a\}$ is decomposable with respect to $\Delta_{1}$ and $\Delta_{2}$, but not decomposable with respect to $\Delta$. Thus, $\left\{\Delta_{1}, \Delta_{2}\right\}$ is not a reduction of $\Delta$.

\section{Related Properties AND PROBlems}

In this subsection, we shall provide a detailed explanation of the relevance of the decomposability verification problem to the problem of supervisor decomposition and the problem of existence of decentralized supervisor. We hope the reader would also be convinced that the decomposability verification problem is a basic problem to investigate the important role played by structures of distributions in determining the decidability and complexity of some basic verification and synthesis problems in decentralized supervisory control ${ }^{18}$.

We need to explain the problem setup in the following. A control constraint $\mathcal{A}$ over $\Sigma$ is a tuple $\left(\Sigma_{S, o}, \Sigma_{S, c}\right)$ of subsets of $\Sigma$. A finite state supervisor ${ }^{19} S$ over $\mathcal{A}$ is a finite state nonmarking automaton over $\Sigma$ with the constraint that at each state

\footnotetext{
${ }^{18}$ This subsection discusses mainly about structural results in the problem of existence of decentralized supervisor; for structural results in decentralized supervisor synthesis problem, the reader is referred to [9].

${ }^{19} \mathrm{We}$ consider non-marking supervisory control [13].
}

of $S: 1)$ there is an outgoing transition labeled by $\sigma$, for each $\left.\sigma \in \Sigma-\Sigma_{S, c} ; 2\right)$ if there is an outgoing transition labeled by $\sigma \in \Sigma-\Sigma_{S, o}$, then this transition is a self-loop. Intuitively, $\Sigma_{S, c}$ is the subset of events supervisor $S$ can disable and $\Sigma_{S, o}$ is the subset of events supervisor $S$ can observe. A decentralized control architecture over $\Sigma$ is just a tuple $\left(\mathcal{A}_{i}\right)_{i=1}^{k}$, where each component $\mathcal{A}_{i}=\left(\Sigma_{S_{i}, o}, \Sigma_{S_{i}, c}\right)$ is a control constraint over $\Sigma$. A tuple $\left(S_{i}\right)_{i=1}^{k}$ of supervisors is said to be over $\left(\mathcal{A}_{i}\right)_{i=1}^{k}$ if each supervisor $S_{i}$ is over $\mathcal{A}_{i}$. Let $\biguplus\left(A_{i}\right)_{i=1}^{k}$ denote the control constraint $\left(\bigcup_{i=1}^{k} \Sigma_{S_{i}, o}, \bigcup_{i=1}^{k} \Sigma_{S_{i}, c}\right)$. We are now ready to state the supervisor decomposition problem and the problem of existence of decentralized supervisor.

Problem 41 (Supervisor Decomposition). Given an arbitrary control constraint $\mathcal{A}$ over $\Sigma$, an arbitrary global finite state supervisor $S$ over $\mathcal{A}$ and an arbitrary decentralized control architecture $\left(\mathcal{A}_{i}\right)_{i=1}^{k}$ over $\Sigma$, where $\mathcal{A}=\biguplus\left(\mathcal{A}_{i}\right)_{i=1}^{k}$, determine the existence of a tuple $\left(S_{i}\right)_{i=1}^{k}$ of local finite state supervisors over $\left(\mathcal{A}_{i}\right)_{i=1}^{k}$ such that $L\left(\|_{i=1}^{k} S_{i}\right)=L(S)$.

Problem 42 (Existence of Decentralized Supervisor). Given an arbitrary finite state automaton $G$ over $\Sigma$, an arbitrary regular language $K$ over $\Sigma$ and an arbitrary decentralized control architecture $\left(\mathcal{A}_{i}\right)_{i=1}^{k}$ over $\Sigma$, determine the existence of a tuple $\left(S_{i}\right)_{i=1}^{k}$ of local finite state supervisors over $\left(\mathcal{A}_{i}\right)_{i=1}^{k}$ such that $G \| S$ is non-blocking and $L_{m}(G \| S)=K$, where $S=\|_{i=1}^{k} S_{i}$.

It is not difficult to observe the following result.

Proposition 43. The prefix-closed decomposability verification problem, where test languages are required to be prefixclosed, is a special case of the supervisor decomposition problem, and the supervisor decomposition problem is a special case of the problem of existence of decentralized supervisor.

Proof: An instance of the decomposability verification problem with prefix-closed test language $L \subseteq \Sigma^{*}$ and distribution $\Delta=\left(\Sigma_{i}\right)_{i=1}^{n}$ is an instance of the supervisor decomposition problem with the control constraint $\mathcal{A}=(\Sigma, \Sigma)$, decentralized control architecture $\left(\mathcal{A}_{i}\right)_{i=1}^{n}=\left(\Sigma_{i}, \Sigma_{i}\right)_{i=1}^{n}$ and global finite state supervisor $S$ over $\mathcal{A}$, where $L(S)=L$. An instance of the supervisor decomposition problem with decentralized control architecture $\left(\mathcal{A}_{i}\right)_{i=1}^{n}$, control constraint $\mathcal{A}=\biguplus\left(\mathcal{A}_{i}\right)_{i=1}^{k}$, and global finite state supervisor $S$ over $\mathcal{A}$ is simply an instance of the problem of existence of decentralized supervisor with $K=L(S)$, decentralized control architecture $\left(\mathcal{A}_{i}\right)_{i=1}^{n}$ and finite state plant $G$, where $L_{m}(G)=L(G)=\Sigma^{*}$.

With the above reduction, we have the following result.

Corollary 44. The supervisor decomposition problem and the problem of existence of decentralized supervisor are PSPACEhard $^{20}$. In particular, the supervisor decomposition problem is PSPACE-hard even when each control constraint $\mathcal{A}_{i}$ satisfies the condition $\Sigma_{S_{i}, o}=\Sigma_{S_{i}, c}=\Sigma_{i}$, where $\left(\Sigma_{1}, \Sigma_{2}, \ldots, \Sigma_{n}\right)$ is a distribution of $\Sigma$; the problem of existence of decentralized supervisor is PSPACE-hard even when $K$ is prefix-closed, $G$ satisfies the condition $L_{m}(G)=L(G)=\Sigma^{*}$ and each control constraint $\mathcal{A}_{i}$ satisfies the condition $\Sigma_{S_{i}, o}=\Sigma_{S_{i}, c}=\Sigma_{i}$, where $\Delta=\left(\Sigma_{1}, \Sigma_{2}, \ldots, \Sigma_{n}\right)$ is a distribution of $\Sigma$.

\footnotetext{
${ }^{20}$ The problem of existence of decentralized supervisor is also in PSPACE (see [16] for an alternative proof of the PSPACE-completeness). Thus, both problems are indeed PSPACE-complete.
} 
Since the prefix-closed decomposability verification problem is a special case of the supervisor decomposition problem, and the supervisor decomposition problem is a special case of the problem of existence of decentralized supervisor, tractable (respectively, intractable) fragments of the first problem can be translated to tractable (respectively, intractable) fragments of the latter two problems. In fact, with the simple reduction provided in Proposition 43, those classes of distributions that render the prefix-closed decomposability verification problem intractable could be interpreted to be classes of decentralized control architectures that render the latter two problems intractable, once we require each local supervisor $S_{i}$ to observe and control the same sub-alphabet $\Sigma_{i}$.

\section{OTHER APPLICATIONS}

In this section, we will present some other applications of the notion of reduction of distributions. Indeed, we will explain some applications of the notion to the problem of computing non-empty decomposable sublanguages (Section VI-A) and the problem of the infimal decomposable superlanguage (Section VI-B), with respect to multiple distributions.

\section{A. Decomposable Sublanguage: Multiple Distributions}

The problem that shall interest us in this subsection is stated below.

Problem 45. Let $P$ be any given set of distributions of $\Sigma$. For an arbitrary regular language $L$ over $\Sigma$, determine the existence of a non-empty sublanguage of $L$ that is decomposable with respect to every distribution in $P$.

The following observation is a straightforward consequence of the notion of reduction of distributions.

Observation 46. Let $P$ be any given set of distributions of $\Sigma$. Suppose $P$ is a reduction of some distribution $\Delta$. Then for an arbitrary language $L$ over $\Sigma, L$ has a non-empty sublanguage that is decomposable with respect to every distribution in $P$ iff $L$ has a non-empty sublanguage that is decomposable with respect to $\Delta$.

We note that Problem 45 is a straightforward generalization of the undecidable decomposable sublanguage problem [8], [9] to multiple distributions. Using Observation 46, we are able to identify a decidable fragment of it using the known characterization result for the decomposable sublanguage problem. We recall that the decomposable sublanguage problem, i.e., the problem of deciding the existence of a non-empty decomposable sublanguage with respect to $\Delta$ for an arbitrary regular language over $\Sigma$, is decidable if and only if $I_{\Delta}$ is transitive [8], [9], which is a consequence of Theorems 2 and 3 of [18].

Theorem 47. Given any set $P$ of distributions of $\Sigma$. Suppose $P$ is a reduction of some distribution $\Delta$. Then the problem of determining the existence of a non-empty sublanguage that is decomposable with respect to every distribution in $P$, for an arbitrary regular language over $\Sigma$, is decidable if and only if $I_{\Delta}$ is transitive.

We use the next example to illustrate Theorem 47.

Example 48. Consider the following two distributions $\Delta_{1}=$ $(\{a, b\},\{a, c, d\})$ and $\Delta_{2}=(\{a, c\},\{a, b, d\})$ of the alphabet $\{a, b, c, d\} .\left\{\Delta_{1}, \Delta_{2}\right\}$ is a reduction of the distribution $\Delta=$ $(\{a, b\},\{a, c\},\{a, d\})$, whose induced independence relation is transitive. Then, according to Theorem 47, it is decidable whether an arbitrary regular language $L$ over $\{a, b, c, d\}$ has a non-empty sublanguage that is decomposable with respect to both distributions $\Delta_{1}$ and $\Delta_{2}$. This result may seem somewhat surprising, for it is undecidable whether an arbitrary regular language $L$ over $\{a, b, c, d\}$ has a non-empty decomposable sublanguage with respect to $\Delta_{1}$ since $\Delta_{1}$ is not transitive (the same conclusion holds for $\Delta_{2}$ ).

\section{B. Decomposable Superlanguage: Multiple Distributions}

In this subsection, we consider the problem of computing the infimal decomposable superlanguage of an arbitrary regular language with respect to all distributions $\Delta_{i}$ 's in any given set $P=\left\{\Delta_{1}, \Delta_{2}, \ldots, \Delta_{n}\right\}$, the existence of which is guaranteed by the fact that the class of decomposable superlanguages is non-empty and closed under arbitrary intersection (see also the proof of Proposition 29, where an instance of this problem incidentally arises; the notion of infimal decomposable superlanguage with respect to a distribution has been mentioned in Proposition 4 of [17]). A straightforward approach to compute the infimal decomposable superlanguage of $L$ is to construct the ascending chain

$$
\begin{gathered}
L \subseteq L^{\Delta_{1}} \subseteq\left(L^{\Delta_{1}}\right)^{\Delta_{2}} \subseteq \ldots \subseteq\left(\left(L^{\Delta_{1}}\right)^{\Delta_{2}} \ldots\right)^{\Delta_{n}} \subseteq \\
\left(\left(\left(L^{\Delta_{1}}\right)^{\Delta_{2}} \ldots\right)^{\Delta_{n}}\right)^{\Delta_{1}} \subseteq \ldots
\end{gathered}
$$

and hopefully the chain would stabilize. Then the supremal element of the chain by construction is the infimal decomposable superlanguage of $L$ with respect to distributions $\Delta_{i}$ 's in $P$. In certain cases, it is possible to directly compute the infimal decomposable superlanguage of $L$. The following proposition is a straightforward consequence of the notion of reduction of distributions.

Proposition 49. Given any set $P$ of distributions. Suppose $P$ is a reduction of some distribution $\Delta$. Then for an arbitrary language $L \subseteq \Sigma^{*}$, the infimal decomposable superlanguage of $L$ with respect to distributions $\Delta_{i}$ 's in $P$ is $L^{\Delta}$.

In particular, if $L$ is regular, then $L^{\Delta}$ is effectively regular.

\section{CONCLUSIONS}

We have provided a detailed study of a notion of reduction of distributions. In particular, we have developed results that can be used to reduce the search space of candidate reductions and used a substitution-based proof technique for reduction verification. In future work, we plan to use Lemma 24 and Lemma 25 for fast production of candidate reductions.

We remark that there are still many open problems left (see for example the list of technical problems in Section III). In particular, we still do not know whether Technical Problem 1 is decidable. It is also of interest to know whether the sound substitution-based proof technique is complete for the problem of reduction verification.

\section{ACKNOWLEDGEMENT}

The authors would like to thank the anonymous reviewers for suggestions and comments that helped improve the paper.

\section{REFERENCES}

[1] Y. Willner, M. Heymann. "Supervisory control of concurrent discrete event systems, Int. Journal of Control, 54(5): 1143-1169, 1991.

[2] F. Lin, W. M. Wonham. "Decentralized supervisory control of discreteevent systems, Information Sciences, 44(3): 199-224, 1988

[3] J. Komenda, T. Masopust, J. H. van Schuppen. "On conditional decomposability", Systems \& Control Letters, 61(12): 1260-1268, 2012.

[4] K. Cai, W. M. Wonham. "Supervisor localization: a top-down approach to distributed control of discrete-event systems", IEEE Trans. of Automatic Control, 55(3): 605-618, 2010. 
[5] K. Rudie, W. M. Wonham. "Think globally, act locally: decentralized supervisory control", IEEE Trans. Automatic Control, 37(11): 1692$1708,1992$.

[6] J. Komenda, T. Masopust. "A bridge between decentralized and coordination control", 51st Annual Allerton Conference on Communication, Control and Computing, pp. 966-972, 2013.

[7] A. Stefanescu. "Automatic synthesis of distributed transition systems", Ph.D. dissertation, University of Stuttgart, 2006.

[8] L. Lin, A. Stefanescu, R. Su, W. Wang, A. R. Shehabinia. "Towards decentralized synthesis: decomposable sublanguage and joint observability problems", American Control Conference, pp. 2047-2052, 2014.

[9] L. Lin, A. Stefanescu, R. Su. "On distributed and parameterized supervisor synthesis problems", IEEE Trans. of Automatic Control, 61(3): 777-782, 2016.

[10] L. Lin, S. Ware, R. Su, W. M. Wonham, "Reduction of distributions and its applications", American Control Conference, In Press, 2017.

[11] J. E. Hopcroft, J. D. Ullman. Introduction to automata theory, languages, and computation, Addison-Wesley, Reading, Massachusetts, 1979.

[12] B. A. Davey, H. A. Priestley. Introduction to lattices and order, Cambridge University Press, 2nd edition, 2002.

[13] W. M. Wonham. Supervisory control of discrete-event systems, Systems Control Group, Dept. of ECE, University of Toronto, Toronto, Canada, 2010.

[14] F. Lei. "Computationally Efficient Supervisor Design for Discrete-Event Systems", Ph.D. dissertation, University of Toronto, 2007.

[15] M. Crouch, N. Immerman, J. Eliot B. Moss. "Finding reductions automatically", Fields of Logic and Computation, pp. 181-200, 2010.

[16] K. Rohloff, S. Lafortune. "PSPACE-completeness of modular supervisory control problems", Discrete Event Dynamic Systems, 15(2): 145$167,2005$.

[17] J. Komenda, H. Marchand, S. Pinchinat. "A constructive and modular approach to decentralized supervisory control problems", 3rd IFAC Workshop on Discrete-Event System Design, 39(17): 111-116, 2006.

[18] J. Sakarovitch. "The "last" decision problem for rational trace languages", LATIN, Vol. 583, pp. 460-473, 1992.

\section{APPENDIX}

The proposed reduction-based approach exploits structures of distributions as a means to achieve complexity reduction for decomposability verification. It is also possible to exploit, in addition, structures of test languages and in particular, we will show an alternative algorithm for testing the decomposability of prefix-closed regular languages. The following proposition is needed, which is a generalization of Lemma 4.1 in [1]. We recall that $I_{\sigma}=\left\{i \in[1, n] \mid \sigma \in \Sigma_{i}\right\}$ for each $\sigma \in \Sigma$.

Proposition 50. Let $L$ be any prefix-closed language over $\Sigma$ and $L_{i}$ any prefix-closed language over $\Sigma_{i}$, for $i \in[1, n]$. If it holds that $L \subseteq \|_{i=1}^{n} L_{i}$, then the following three statements are equivalent:

1) $L \neq \|_{i=1}^{n} L_{i}$.

2) $\exists \sigma \in \Sigma, \exists s \in L, s \sigma \notin L \wedge\left(\forall i \in I_{\sigma}, P_{i}(s \sigma) \in L_{i}\right)$

3) $\exists \sigma \in \Sigma,\left(L \sigma \cap L^{c}\right) \cap\left(\bigcap_{i \in I_{\sigma}} P_{i}^{-1}\left(L_{i}\right)\right) \neq \emptyset$

We still need the next result, which is straightforward.

Lemma 51. For any prefix-closed regular language $L \subseteq \Sigma^{*}$ that is given by a deterministic finite automaton $M, L \sigma \cap L^{c}$ is linear time computable from $M$, for each $\sigma \in \Sigma$.

From Proposition 50 and Lemma 51, we have the following.

Corollary 52. For any prefix-closed regular language $L \subseteq \Sigma^{*}$ that is given by a deterministic finite automaton of state size $m$, the complexity of verifying the decomposability of $L$ with respect to $\Delta$ is $\mathcal{O}\left(\sum_{\sigma \in \Sigma}\left(\left(\left|I_{\sigma}\right|+1\right)|\Sigma|-\sum_{i \in I_{\sigma}}\left|\Sigma_{i}\right|\right) m^{\left|I_{\sigma}\right|+1}\right)$.

Remark 53. Suppose $\max _{\sigma \in \Sigma}\left|I_{\sigma}\right|=k$, then a crude upper bound for the above time complexity is $\mathcal{O}\left(k|\Sigma|^{2} m^{k+1}\right)$.

It turns out that verifying $L=\|_{i=1}^{n} P_{i}(L)$ costs $\mathcal{O}\left(|\Sigma|^{2} m^{2}\right)$ when the sub-alphabets of $\Delta$ are pairwise disjoint and $L$ is a prefix-closed regular language. Since usually $|\Sigma| \ll m$, this is an improvement of the complexity $\mathcal{O}\left(n|\Sigma| m^{3}\right)$ needed for the reduction-based approach. However, in general, the reductionbased approach and Proposition 50 are not comparable. This is illustrated by the next example.

Example 54. Consider the alphabet $\Sigma=\{a, b, c, d, e, f\}$ and the distribution $\Delta=(\{a, b, c\},\{b, c, d\},\{c, d, e\},\{d, e, f\})$ of $\Sigma$. Clearly, $\left\{\Delta_{1}, \Delta_{2}, \Delta_{3}\right\}$ is a reduction of $\Delta$, where

$$
\left\{\begin{aligned}
\Delta_{1} & =(\{a, b, c, d\},\{c, d, e, f\}) \\
\Delta_{2} & =(\{a, b, c\},\{b, c, d, e, f\}) \\
\Delta_{3} & =(\{a, b, c, d, e\},\{d, e, f\})
\end{aligned}\right.
$$

Thus, using the reduction-based approach, we can verify the decomposability of $L$ with respect to $\Delta$ in $\mathcal{O}\left(\mathrm{m}^{3}\right)$ time. The approach based on Proposition 50 costs $\mathcal{O}\left(\mathrm{m}^{4}\right)$.

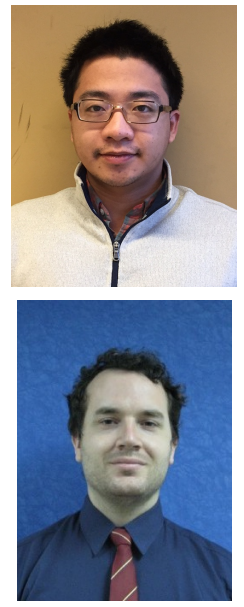

Liyong Lin received the $\mathrm{BE}$ degree and $\mathrm{PhD}$ degree in electrical engineering in 2011 and 2016, respectively, both from Nanyang Technological University, where he has also worked as a project officer. Since June 2016, he has been a postdoctoral fellow in the University of Toronto. His main research interests include supervisory control theory and formal methods. He previously was an undergraduate intern in Data Storage Institute, where he worked on single and dual-stage servomechanism of hard disk drives.

Simon Ware received his Bachelor of Computing and Mathematical Sciences degree with Honours from the University of Waikato in Hamilton, New Zealand in 2007. Also in 2007, he was involved in a project for discrete event simulation of port biosecurity procedures at AgResearch in Hamilton. $\mathrm{He}$ received his Ph.D. in Computer Science from the University of Waikato in 2014. He is currently a research fellow at Nanyang Technological University in Singapore. His main research interests are liveness and fairness properties of discrete event systems.

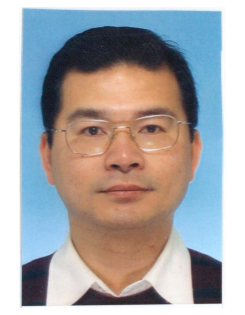

Rong Su (M'11-SM'14) received the BE degree in Automatic Control from the University of Science and Technology of China in 1997, and the MASc and PhD degrees both in electrical engineering from the University of Toronto in 2000 and 2004, respectively. Since then he was affiliated with University of Waterloo and Technical University of Eindhoven before he joined Nanyang Technological University in 2010. His research interests include discrete event systems, supervisory control, model-based fault diagnosis, multi-agent systems, optimization and scheduling with applications in green buildings, flexible manufacturing, power management and intelligent transportation systems. In the aforementioned areas, he has over 100 publications in journals, book chapters, and conference proceedings, and two patents. Dr Su is an Associate Editor for Journal of Control and Decision, and Transactions of the Institute of Measurement and Control, and the Chair of IEEE Control Systems Society Technical Committee on Smart Cities.

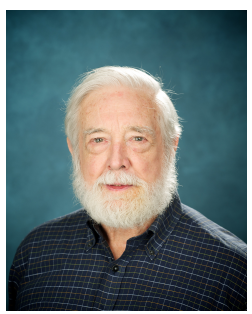

W. M. Wonham received the B. Eng. degree in engineering physics from McGill University in1956, and the Ph.D. in control engineering from the University of Cambridge (U.K.) in 1961. From 1961 to 1969 he was associated with several U.S. research groups in control. Since 1970 he has been a faculty member in Systems Control, with the Department of Electrical and Computer Engineering of the University of Toronto. Wonhams research interests have included stochastic control and filtering, geometric multivariable control, and discrete-event systems. $\mathrm{He}$ is the author of "Linear Multivariable Control: A Geometric Approach" (Springer- Verlag: 3rd ed. 1985), co-author (with C. Ma) of "Nonblocking Supervisory Control of State Tree Structures (Springer-Verlag: 2005), and coauthor (with K. Cai) of "Supervisor Localization: A Top-Down Approach to Distributed Control of Discrete-Event Systems" (Springer-Verlag: 2015).

Wonham is a Fellow of the Royal Society of Canada, a Life Fellow of the IEEE, a Foreign Member of the (U.S.) National Academy of Engineering, and an Honorary Professor of the Beijing University of Aeronautics and Astronautics. In 1987 he received the IEEE Control Systems Science and Engineering Award and in 1990 was Brouwer Medallist of the Netherlands Mathematical Society. In 1996 he was appointed University Professor in the University of Toronto, and in 2000 University Professor Emeritus. 